Pacific

Journal of

Mathematics

REIDEMEISTER TORSION, THE THURSTON NORM AND HARVEY'S INVARIANTS

STEFAN FRIEDL

Volume $230 \quad$ No. 2

April 2007 


\title{
REIDEMEISTER TORSION, THE THURSTON NORM AND HARVEY'S INVARIANTS
}

\author{
STEFAN FRIEDL
}

\begin{abstract}
Cochran introduced Alexander polynomials over noncommutative Laurent polynomial rings. Their degrees were studied by Cochran, Harvey and Turaev, who gave lower bounds on the Thurston norm. We extend Cochran's definition to twisted Alexander polynomials, and show how Reidemeister torsion relates to these invariants, giving lower bounds on the Thurston norm in terms of the Reidemeister torsion. This yields a concise formulation of the bounds of Cochran, Harvey and Turaev. The Reidemeister torsion approach also provides a natural approach to proving and extending certain monotonicity results of Cochran and Harvey.
\end{abstract}

\section{Introduction}

The following algebraic setup allows us to define twisted noncommutative Alexander polynomials. Let $\mathbb{K}$ be a (skew) field and $\gamma: \mathbb{K} \rightarrow \mathbb{K}$ a ring homomorphism. Denote by $\mathbb{K}_{\gamma}\left[t^{ \pm 1}\right]$ the skew Laurent polynomial ring over $\mathbb{K}$, so the elements in $\mathbb{K}_{\gamma}\left[t^{ \pm 1}\right]$ are formal sums $\sum_{i=m}^{n} a_{i} t^{i}(m \leq n \in \mathbb{Z})$ with $a_{i} \in \mathbb{K}$. Addition is given by addition of the coefficients, and multiplication is defined using the rule $t^{i} a=\gamma^{i}(a) t^{i}$ for any $a \in \mathbb{K}$.

Let $X$ be a connected CW complex with finitely many cells in dimension $i$. Given a representation $\alpha: \pi_{1}(X) \rightarrow \mathrm{GL}\left(\mathbb{K}_{\gamma}\left[t^{ \pm 1}\right], d\right)$ we can consider the $\mathbb{K}_{\gamma}\left[t^{ \pm 1}\right]$ modules $H_{i}^{\alpha}\left(X ; \mathbb{K}_{\gamma}\left[t^{ \pm 1}\right]^{d}\right)$ and we define twisted noncommutative Alexander polynomials $\Delta_{i}^{\alpha}(t) \in \mathbb{K}_{\gamma}\left[t^{ \pm 1}\right]$ (see Section 3.3 for details). Twisted Alexander polynomials over commutative Laurent polynomial rings were first introduced in [Lin 2001], Alexander polynomials over skew Laurent polynomial rings in [Cochran 2004]. Our definition is a combination of the definitions in [Kirk and Livingston 1999] and [Cochran 2004]. In Theorem 3.1 we describe the indeterminacy of these polynomials.

Denote by $\mathbb{K}_{\gamma}(t)$ the quotient field of $\mathbb{K}_{\gamma}\left[t^{ \pm 1}\right]$. We denote the induced representation $\pi_{1}(X) \rightarrow \operatorname{GL}\left(\mathbb{K}_{\gamma}\left[t^{ \pm 1}\right], d\right) \rightarrow \operatorname{GL}\left(\mathbb{K}_{\gamma}(t), d\right)$ by $\alpha$ as well. If the homology groups $H_{i}^{\alpha}\left(X ; \mathbb{K}_{\gamma}(t)^{d}\right)$ vanish and if $X$ is a finite connected CW complex, then

MSC2000: primary 57M27; secondary 57N10.

Keywords: Thurston norm, Reidemeister torsion, 3-manifolds, knot genus. 
we can define the Reidemeister torsion $\tau(X, \alpha) \in K_{1}\left(\mathbb{K}_{\gamma}(t)\right) / \pm \alpha\left(\pi_{1}(X)\right)$. An important tool is the Dieudonné determinant, which defines an isomorphism

$$
\operatorname{det}: K_{1}\left(\mathbb{K}_{\gamma}(t)\right) \rightarrow \mathbb{K}_{\gamma}(t)_{a b}^{\times},
$$

where $\mathbb{K}_{\gamma}(t)_{a b}^{\times}$denotes the abelianization of the multiplicative group $\mathbb{K}_{\gamma}(t)^{\times}=$ $\mathbb{K}_{\gamma}(t) \backslash\{0\}$. We can therefore study $\operatorname{det} \tau(X, \alpha) \in \mathbb{K}_{\gamma}(t)_{a b}^{\times} / \pm \operatorname{det} \alpha\left(\pi_{1}(X)\right)$. We refer to Sections 2.3 and 3.1 for details.

The following result generalizes commutative results of Turaev [1986; 2001] and Kirk and Livingston [1999].

Theorem 1.1. Let $X$ be a finite connected $C W$ complex of dimension $n$. Let $\alpha$ : $\pi_{1}(X) \rightarrow \mathrm{GL}\left(\mathbb{K}_{\gamma}\left[t^{ \pm 1}\right], d\right)$ be a representation such that $H_{*}^{\alpha}\left(X ; \mathbb{K}_{\gamma}(t)^{d}\right)=0$. Then $\Delta_{i}^{\alpha}(t) \neq 0$ for all $i$ and

$$
\operatorname{det} \tau(X, \alpha)=\prod_{i=0}^{n-1} \Delta_{i}^{\alpha}(t)^{(-1)^{i+1}} \in \mathbb{K}_{\gamma}(t)_{a b}^{\times} /\left\{k t^{e} \mid k \in \mathbb{K} \backslash\{0\}, e \in \mathbb{Z}\right\} .
$$

For $f(t)=\sum_{i=m}^{n} a_{i} t^{i} \in \mathbb{K}_{\gamma}\left[t^{ \pm 1}\right] \backslash\{0\}$ with $a_{m} \neq 0, a_{n} \neq 0$, we define its degree to be deg $f(t)=n-m$. We can extend this to a degree function deg: $\mathbb{K}_{\gamma}(t) \backslash\{0\} \rightarrow$ $\mathbb{Z}$. We denote $\operatorname{deg} \operatorname{det} \tau(X, \alpha)$ by $\operatorname{deg} \tau(X, \alpha)$. Theorem 1.1 then implies that the degree of $\tau(X, \alpha)$ is the alternating sum of the degrees of the twisted Alexander polynomials (see Corollary 3.6).

We now turn to the study of 3-manifolds. Here and throughout the paper we will assume that all manifolds are compact, orientable and connected. Recall that given a 3-manifold $M$ and $\phi \in H^{1}(M ; \mathbb{Z})$ the Thurston norm [1986] of $\phi$ is defined as

$$
\|\phi\|_{T}=\min \{-\chi(\hat{S}) \mid S \subset M \text { properly embedded surface dual to } \phi\}
$$

where $\hat{S}$ denotes the result of discarding all connected components of $S$ with positive Euler characteristic. As an example, consider $X(K)=S^{3} \backslash v K$, where $K \subset S^{3}$ is a knot and $\nu K$ denotes an open tubular neighborhood of $K$ in $S^{3}$. Let $\phi \in H^{1}(X(K) ; \mathbb{Z})$ be a generator, then $\|\phi\|_{T}=2$ genus $(K)-1$.

Let $X$ be a connected CW complex and let $\phi \in H^{1}(X ; \mathbb{Z})$. We identify henceforth $H^{1}(X ; \mathbb{Z})$ with $\operatorname{Hom}\left(H_{1}(X ; \mathbb{Z}), \mathbb{Z}\right)$ and $\operatorname{Hom}\left(\pi_{1}(X), \mathbb{Z}\right)$. A representation $\alpha: \pi_{1}(X) \rightarrow \mathrm{GL}\left(\mathbb{K}_{\gamma}\left[t^{ \pm 1}\right], d\right)$ is called $\phi$-compatible if for any $g \in \pi_{1}(X)$ we have $\alpha(g)=A t^{\phi(g)}$ for some $A \in \mathrm{GL}(\mathbb{K}, d)$. This generalizes a notion of Turaev [2002a].

The following theorem gives lower bounds on the Thurston norm using Reidemeister torsion. It contains the lower bounds of McMullen [2002], Cochran [2004], Harvey [2005], Turaev [2002a] and of the paper [Friedl and Kim 2006]. To our knowledge this theorem is the strongest of its kind. Not only does it contain these results, the formulation of the inequalities given in the papers just cited in terms 
of the degrees of Reidemeister torsion also gives a very concise reformulation of their results.

Theorem 1.2. Let $M$ be a 3-manifold with empty or toroidal boundary. Let $\phi \in$ $H^{1}(M ; \mathbb{Z})$ and $\alpha: \pi_{1}(M) \rightarrow \mathrm{GL}\left(\mathbb{K}_{\gamma}\left[t^{ \pm 1}\right], d\right)$ a $\phi$-compatible representation. Then $\tau(M, \alpha)$ is defined if and only if $\Delta_{1}^{\alpha}(t) \neq 0$. If $\tau(M, \alpha)$ is defined, then

$$
\|\phi\|_{T} \geq \frac{1}{d} \operatorname{deg} \tau(M, \alpha) .
$$

If $(M, \phi)$ fibers over $S^{1}$, then

$$
\|\phi\|_{T}=\max \left\{0, \frac{1}{d} \operatorname{deg} \tau(M, \alpha)\right\} .
$$

The most commonly used skew fields are the quotient fields $\mathbb{K}(G)$ of group rings $\mathbb{F}[G]$ ( $\mathbb{F}$ a commutative field) for certain torsion-free groups $G$, we refer to Section 5.1 for details. The following theorem says roughly that larger groups give better bounds on the Thurston norm. The main idea of the proof is to use the fact that Reidemeister torsion behaves well under ring homomorphisms, in contrast to Alexander polynomials. See Section 6 or [Harvey 2006] for the definition of an admissible triple.

Theorem 1.3. Let $M$ be a 3-manifold with empty or toroidal boundary or let $M$ be a 2-complex with $\chi(M)=0$. Let $\phi \in H^{1}(M ; \mathbb{Z})$. Let $\alpha: \pi_{1}(M) \rightarrow \operatorname{GL}(\mathbb{F}, d)$, $\mathbb{F}$ a commutative field, be a representation and $\left(\varphi_{G}: \pi \rightarrow G, \varphi_{H}: \pi \rightarrow H, \phi\right)$ an admissible triple for $\pi_{1}(M)$, in particular we have epimorphisms $G \rightarrow H \rightarrow \mathbb{Z}$. Write $G^{\prime}=\operatorname{Ker}\{G \rightarrow \mathbb{Z}\}$ and $H^{\prime}=\operatorname{Ker}\{H \rightarrow \mathbb{Z}\}$.

If $\tau\left(M, \varphi_{H} \otimes \alpha\right) \in K_{1}\left(\mathbb{K}\left(H^{\prime}\right)(t)\right)$ is defined, then $\tau\left(M, \varphi_{G} \otimes \alpha\right) \in K_{1}\left(\mathbb{K}\left(G^{\prime}\right)(t)\right)$ is defined. Furthermore in that case

$$
\operatorname{deg} \tau\left(M, \varphi_{G} \otimes \alpha\right) \geq \operatorname{deg} \tau\left(M, \varphi_{H} \otimes \alpha\right) .
$$

A similar theorem holds for 2-complexes with Euler characteristic zero. As a special case consider the case that $\alpha$ is the trivial representation. Using Theorem 1.1 we can recover the monotonicity results of [Cochran 2004; Harvey 2006]. We hope that our alternative proof using Reidemeister torsion will contribute to the understanding of their results.

In the next section we recall the definition of Reidemeister torsion. In Section 3 we introduce twisted noncommutative Alexander polynomials, compute their indeterminacies in Theorem 3.1 and prove Theorem 1.1. Beginning with Section 4 we concentrate on 3-manifolds: Section 4 gives the proof of Theorem 1.2, Section 5 contains examples of $\phi$-compatible representations, in Section 6 we prove Theorem 1.3, and in Section 7 we show that it implies Cochran's and Harvey's monotonicity results. We conclude with a few open questions in Section 8. 


\section{Reidemeister torsion}

2.1. Definition of $K_{1}(R)$. For the remainder of the paper we will only consider associative rings $R$ with $1 \neq 0$ and with the property that if $r \neq s \in \mathbb{N}_{0}$, then $R^{r}$ is not isomorphic to $R^{s}$ as an $R$-module.

For such a ring $R$ define $\operatorname{GL}(R)=\underline{\lim } \operatorname{GL}(R, d)$, where the maps $\operatorname{GL}(R, d) \rightarrow$ $\mathrm{GL}(R, d+1)$ in the direct system are given by $A \mapsto\left(\begin{array}{cc}A & 0 \\ 0 & 1\end{array}\right)$. Then $K_{1}(R)$ is defined as $\mathrm{GL}(R) /[\mathrm{GL}(R), \mathrm{GL}(R)]$. Note that $K_{1}(R)$ is an abelian group. For details we refer to [Milnor 1966; Turaev 2001]. There exists a canonical map $\mathrm{GL}(R, d) \rightarrow K_{1}(R)$ for every $d$, in particular there exists a homomorphism from the units of $R$ into $K_{1}(R)$. By abuse of notation we denote the image of $A \in \mathrm{GL}(R, d)$ in $K_{1}(R)$ by $A$ as well. We denote by $-A$ the product of $A \in K_{1}(R)$ by the image of $(-1)$ under the map $\operatorname{GL}(R, 1) \rightarrow K_{1}(R)$.

We will often make use of the observation (see [Rosenberg 1994, p. 61]) that for $A \in \mathrm{GL}\left(R, d_{1}\right), B \in \mathrm{GL}\left(R, d_{2}\right)$ the product $A B \in K_{1}(R)$ is given by

$$
A B=\left(\begin{array}{cc}
A & 0 \\
0 & B
\end{array}\right) \in K_{1}(R) .
$$

2.2. Definition of Reidemeister torsion. Let $C_{*}$ be a finite free chain complex of $R$-modules. By this we mean a chain complex of free finite right $R$-modules such that $C_{i}=0$ for all but finitely many $i \in \mathbb{Z}$. Let $\mathscr{C}_{i} \subset C_{i}$ be a basis for all $i$ with $C_{i} \neq 0$. Assume that $B_{i}=\operatorname{Im}\left(C_{i+1}\right) \subset C_{i}$ is free, pick a basis $\mathscr{B}_{i}$ of $B_{i}$ and a lift $\tilde{\mathscr{B}}_{i}$ of $\mathscr{B}_{i}$ to $C_{i+1}$. We write $\mathscr{B}_{i} \tilde{\mathscr{P}}_{i-1}$ for the collection of elements given by $\mathscr{B}_{i}$ and $\tilde{\mathscr{P}}_{i-1}$. Since $C_{*}$ is acyclic this is indeed a basis for $C_{i}$. Then we define the Reidemeister torsion of the based acyclic complex $\left(C_{*},\left\{\mathscr{C}_{i}\right\}\right)$ to be

$$
\tau\left(C_{*},\left\{\mathscr{C}_{i}\right\}\right)=\prod\left[\mathscr{B}_{i} \tilde{\mathscr{B}}_{i-1} / \mathscr{C}_{i}\right]^{(-1)^{i+1}} \in K_{1}(R),
$$

where $[d / e]$ denotes the matrix of a basis change, i.e. $[d / e]=\left(a_{i j}\right)$ where $d_{i}=$ $\sum_{j} a_{j i} e_{j}$. (In contrast to Turaev's book we view vectors as column vectors, so our matrix is the transpose of the matrix in [Turaev 2001, p. 1].)

It is easy to see that $\tau\left(C_{*},\left\{\mathscr{C}_{i}\right\}\right)$ is independent of the choice of $\left\{\mathscr{B}_{i}\right\}$ and of the choice of the lifts $\tilde{\mathscr{P}}_{i}$. If the $R$-modules $B_{i}$ are not free, then one can show that they are stably free and a stable basis will then make the definition work again. See [Milnor 1966, p. 369] or [Turaev 2001, p. 13] for details.

2.3. Reidemeister torsion of a $\mathbf{C W}$ complex. Let $X$ be a connected $\mathrm{CW}$ complex. Denote the universal cover of $X$ by $\tilde{X}$. We view $C_{*}(\tilde{X})$, the chain complex of the universal cover, as a chain complex of right $\mathbb{Z}\left[\pi_{1}(X)\right]$-modules, where the $\mathbb{Z}\left[\pi_{1}(X)\right]$-module structure is given via deck transformations.

Let $R$ be a ring. Let $\alpha: \pi_{1}(X) \rightarrow \operatorname{GL}(R, d)$ be a representation. This equips $R^{d}$ with a left $\mathbb{Z}\left[\pi_{1}(X)\right]$-module structure. We can therefore consider the chain 
complex $C_{*}^{\alpha}\left(X ; R^{d}\right)=C_{*}(\tilde{X}) \otimes_{\mathbb{Z}\left[\pi_{1}(X)\right]} R^{d}$. This is a finite free chain complex of (right) $R$-modules. We denote its homology by $H_{*}^{\alpha}\left(X ; R^{d}\right)$, we drop $\alpha$ from the notation if it is clear from the context.

Now assume that $X$ is a finite connected CW complex. If

$$
H_{i}^{\alpha}\left(X ; R^{d}\right)=H_{i}\left(C_{*}^{\alpha}\left(X ; R^{d}\right)\right) \neq 0
$$

for some $i$, we write $\tau(X, \alpha)=0$. Otherwise denote the $i$-cells of $X$ by $\sigma_{i}^{1}, \ldots, \sigma_{i}^{r_{i}}$ and denote by $e_{1}, \ldots, e_{d}$ the standard basis of $R^{d}$. Pick an orientation for each cell $\sigma_{i}^{j}$, and also pick a lift $\tilde{\sigma}_{i}^{j}$ for each cell $\sigma_{i}^{j}$ to the universal cover $\tilde{X}$. We get a basis

$$
\mathscr{C}_{i}=\left\{\tilde{\sigma}_{i}^{1} \otimes e_{1}, \ldots, \tilde{\sigma}_{i}^{1} \otimes e_{d}, \ldots, \tilde{\sigma}_{i}^{r_{i}} \otimes e_{1}, \ldots, \tilde{\sigma}_{i}^{r_{i}} \otimes e_{d}\right\}
$$

for $C_{i}^{\alpha}\left(X ; R^{d}\right)$. Then we can define

$$
\tau\left(C_{*}^{\alpha}\left(X ; R^{d}\right),\left\{\mathscr{C}_{i}\right\}\right) \in K_{1}(R) .
$$

This element depends only on the ordering and orientation of the cells and on the choice of lifts of the cells to the universal cover. Therefore

$$
\tau(X, \alpha)=\tau\left(C_{*}^{\alpha}\left(X, R^{d}\right),\left\{\mathscr{C}_{i}\right\}\right) \in K_{1}(R) / \pm \alpha\left(\pi_{1}(X)\right)
$$

is a well defined invariant of the CW complex $X$.

Now let $M$ be a compact PL manifold. Pick any finite CW structure for $M$ to define $\tau(M, \alpha) \in K_{1}(R) / \pm \alpha\left(\pi_{1}(M)\right)$. By Chapman's theorem [1974] this is a well defined invariant of the manifold (independent of the choice of the $\mathrm{CW}$ structure).

2.4. Computation of Reidemeister torsion. We explain an algorithm for computing Reidemeister torsion formulated in [Turaev 2001, Section 2.1] in the commutative case.

Assume that we have a finite free chain complex of $R$-modules

$$
0 \rightarrow C_{m} \stackrel{\partial_{m}}{\rightarrow} C_{m-1} \rightarrow \cdots \rightarrow C_{1} \stackrel{\partial_{1}}{\rightarrow} C_{0} \rightarrow 0 .
$$

Let $A_{i}=\left(a_{j k}^{i}\right)$ be the matrix representing $\partial_{i}$ corresponding to the given bases. Again, in contrast with Turaev, we view the elements in $R^{\operatorname{rank}\left(C_{i}\right)}$ as column vectors.

Following [Turaev 2001, p. 8] we define a matrix chain for $C$ to be a collection of sets $\xi=\left(\xi_{0}, \xi_{1}, \ldots, \xi_{m}\right)$ where $\xi_{i} \subset\left\{1,2, \ldots, \operatorname{rank}\left(C_{i}\right)\right\}$ so that $\xi_{0}=\varnothing$. Given a matrix chain $\xi$ we define $A_{i}(\xi), i=1, \ldots, m$ to be the matrix formed by the entries $a_{j k}^{i}$ with $j \notin \xi_{i-1}$ and $k \in \xi_{i}$. Put differently the matrix $\left(a_{j k}^{i}\right)_{j k}$ is given by considering only the $\xi_{i}$-columns of $A_{i}$ and with the $\xi_{i-1}$-rows removed.

We say that a matrix chain $\xi$ is a $\tau$-chain if $A_{1}(\xi), \ldots, A_{m}(\xi)$ are square matrices. The following is the generalization of Turaev's Theorem 2.2 to the noncommutative setting. His proof can easily be generalized to this more general setting. 
Theorem 2.1. Let $\xi$ be a $\tau$-chain such that $A_{i}(\xi)$ is invertible for all odd $i$. Then $A_{i}(\xi)$ is invertible for all even $i$ if and only if $H_{*}(C)=0$. If $H_{*}(C)=0$, then

$$
\tau(C)=\epsilon \prod_{i=1}^{m} A_{i}(\xi)^{(-1)^{i}} \in K_{1}(R) \quad \text { for some } \epsilon \in\{ \pm 1\} .
$$

This proposition is the reason why Reidemeister torsion behaves in general well under ring homomorphisms.

\section{Reidemeister torsion and Alexander polynomials}

3.1. Laurent polynomial rings and the Dieudonné determinant. For the remainder of this paper let $\mathbb{K}$ be a (skew) field and let $\mathbb{K}_{\gamma}\left[t^{ \pm 1}\right]$ be a skew Laurent polynomial ring. By [Dodziuk et al. 2003, Corollary 6.3] the ring $\mathbb{K}_{\gamma}\left[t^{ \pm 1}\right]$ has a classical quotient field $\mathbb{K}_{\gamma}(t)$ which is flat over $\mathbb{K}_{\gamma}\left[t^{ \pm 1}\right]$ (compare [Ranicki 1998, p. 99]). In particular we can view $\mathbb{K}_{\gamma}\left[t^{ \pm 1}\right]$ as a subring of $\mathbb{K}_{\gamma}(t)$ and any element in $\mathbb{K}_{\gamma}(t)$ is of the form $f(t) g(t)^{-1}$ for some $f(t) \in \mathbb{K}_{\gamma}\left[t^{ \pm 1}\right]$ and $g(t) \in \mathbb{K}_{\gamma}\left[t^{ \pm 1}\right] \backslash\{0\}$. We refer to Theorem 5.1 for a related result. Recall that we write $\mathbb{K}_{\gamma}(t)^{\times}=\mathbb{K}_{\gamma}(t) \backslash\{0\}$.

In the following we mean by an elementary column (row) operation the addition of a right multiple (left multiple) of one column (row) to a different column (row). Let $A$ be an invertible $k \times k$ matrix over the skew field $\mathbb{K}_{\gamma}(t)$. After elementary row operations we can turn $A$ into a diagonal matrix $D=\left(d_{i j}\right)$. Then the Dieudonné determinant $\operatorname{det} A \in \mathbb{K}_{\gamma}(t)_{a b}^{\times}=\mathbb{K}_{\gamma}(t)^{\times} /\left[\mathbb{K}_{\gamma}(t)^{\times}, \mathbb{K}_{\gamma}(t)^{\times}\right]$is defined to be $\prod_{i=1}^{k} d_{i i}$. This is a well defined map. The Dieudonné determinant is invariant under elementary row and column operations, and induces an isomorphism $\operatorname{det}: K_{1}\left(\mathbb{K}_{\gamma}(t)\right) \rightarrow \mathbb{K}_{\gamma}(t)_{a b}^{\times}$. Using the last observation in Section 2.1 it is easy to see that $A=\operatorname{det} A \in K_{1}\left(\mathbb{K}_{\gamma}(t)\right)$. We will often make use of this equality. We refer to [Rosenberg 1994, Theorem 2.2.5 and Corollary 2.2.6] for more details.

In the introduction we defined deg: $\mathbb{K}_{\gamma}\left[t^{ \pm 1}\right] \backslash\{0\} \rightarrow \mathbb{N}$. This can be extended to a homomorphism deg: $\mathbb{K}_{\gamma}(t)^{\times} \rightarrow \mathbb{Z}$ via $\operatorname{deg}\left(f(t) g(t)^{-1}\right)=\operatorname{deg} f(t)-\operatorname{deg} g(t)$ for $f(t), g(t) \in \mathbb{K}_{\gamma}\left[t^{ \pm 1}\right] \backslash\{0\}$. Clearly the degree map vanishes on $\left[\mathbb{K}_{\gamma}(t)^{\times}, \mathbb{K}_{\gamma}(t)^{\times}\right]$ and we get an induced homomorphism $K_{1}\left(\mathbb{K}_{\gamma}(t)\right) \rightarrow \mathbb{K}_{\gamma}(t)_{a b}^{\times} \rightarrow \mathbb{Z}$ which we also denote by deg.

3.2. Orders of $\mathbb{K}_{\gamma}\left[t^{ \pm 1}\right]$-modules. Let $H$ be a finitely generated right $\mathbb{K}_{\gamma}\left[t^{ \pm 1}\right]$ module. The ring $\mathbb{K}_{\gamma}\left[t^{ \pm 1}\right]$ is a principal ideal domain (PID) since $\mathbb{K}$ is a skew field. We can therefore find an isomorphism

$$
H \cong \bigoplus_{i=1}^{l} \mathbb{K}_{\gamma}\left[t^{ \pm 1}\right] / p_{i}(t) \mathbb{K}_{\gamma}\left[t^{ \pm 1}\right]
$$

for $p_{i}(t) \in \mathbb{K}_{\gamma}\left[t^{ \pm 1}\right]$ for $i=1, \ldots, l$. Following [Cochran 2004] we define ord $(H)=$ $\prod_{i=1}^{l} p_{i}(t) \in \mathbb{K}_{\gamma}\left[t^{ \pm 1}\right]$. This is called the order of $H$. 
Note that $\operatorname{ord}(H) \in \mathbb{K}_{\gamma}\left[t^{ \pm 1}\right]$ has a high degree of indeterminacy. For example writing the $p_{i}(t)$ in a different order will change ord $(H)$. Furthermore we can change $p_{i}(t)$ by multiplication by any element of the form $k t^{e}$ where $k \in \mathbb{K}^{\times}=$ $\mathbb{K} \backslash\{0\}$ and $e \in \mathbb{Z}$. The following theorem can be viewed as saying that these are all possible indeterminacies.

Theorem 3.1. Let $H$ be a finitely generated right $\mathbb{K}_{\gamma}\left[t^{ \pm 1}\right]$-module. Then $\operatorname{ord}(H)=$ 0 if and only if $H$ is not $\mathbb{K}_{\gamma}\left[t^{ \pm 1}\right]$-torsion. If $\operatorname{ord}(H) \neq 0$, then $\operatorname{ord}(H) \in \mathbb{K}_{\gamma}\left[t^{ \pm 1}\right]$ is well defined considered as an element in $\mathbb{K}_{\gamma}(t)_{a b}^{\times}$up to multiplication by an element of the form $k t^{e}, k \in \mathbb{K}^{\times}$and $e \in \mathbb{Z}$.

The first statement is clear. We postpone the proof of the second statement of the theorem to Section 3.4. We refer to [Cochran 2004, p. 367] for an alternative discussion of the indeterminacy of $\operatorname{ord}(H)$; the idea of considering $\operatorname{ord}(H)$ as an element in $\mathbb{K}_{\gamma}(t)_{a b}^{\times}$is already present there.

It follows from Theorem 3.1 that $\operatorname{deg} \operatorname{ord}(H)$ is well defined. In fact we have the following interpretation of ord $(H)$.

Lemma 3.2 [Cochran 2004, p. 368]. Let $H$ be a finitely generated right $\mathbb{K}_{\gamma}\left[t^{ \pm 1}\right]$ torsion module. Then

$$
\operatorname{deg} \operatorname{ord}(H)=\operatorname{dim}_{\mathbb{K}} H .
$$

Here we used that by [Stenström 1975, Proposition I.2.3] and [Cohn 1985, p. 48] every right $\mathbb{K}$-module $V$ is free and has a well defined $\operatorname{dimension}_{\operatorname{dim}} \mathbb{\ll}(V)$.

Proof. It is easy to see that for $f(t) \in \mathbb{K}_{\gamma}\left[t^{ \pm 1}\right] \backslash\{0\}$ we have

$$
\operatorname{deg} f(t)=\operatorname{dim}_{\mathbb{K}}\left(\mathbb{K}_{\gamma}\left[t^{ \pm 1}\right] / f(t) \mathbb{K}_{\gamma}\left[t^{ \pm 1}\right]\right) .
$$

The lemma is now immediate.

3.3. Alexander polynomials. Let $X$ be a connected CW complex with finitely many cells in dimension $i$. Let $\alpha: \pi_{1}(X) \rightarrow \mathrm{GL}\left(\mathbb{K}_{\gamma}\left[t^{ \pm 1}\right], d\right)$ be a representation. The right $\mathbb{K}_{\gamma}\left[t^{ \pm 1}\right]$-module $H_{i}\left(X ; \mathbb{K}_{\gamma}\left[t^{ \pm 1}\right]^{d}\right)$ is called twisted (noncommutative) Alexander module. Similar modules were studied in [Cochran 2004; Harvey 2005; Turaev 2002a]. Note that $H_{i}\left(X ; \mathbb{K}_{\gamma}\left[t^{ \pm 1}\right]^{d}\right)$ is a finitely generated $\mathbb{K}_{\gamma}\left[t^{ \pm 1}\right]$-module since we assumed that $X$ has only finitely many cells in dimension $i$ and since $\mathbb{K}_{\gamma}\left[t^{ \pm 1}\right]$ is a PID. We now define $\Delta_{i}^{\alpha}(t)=\operatorname{ord}\left(H_{i}\left(X ; \mathbb{K}_{\gamma}\left[t^{ \pm 1}\right]^{d}\right)\right) \in \mathbb{K}_{\gamma}\left[t^{ \pm 1}\right]$, this is called the (twisted) $i$-th Alexander polynomial of $(X, \alpha)$.

The degrees of these polynomials (corresponding to one-dimensional representations) have been studied recently in various contexts [Cochran 2004; Harvey 2005; 2006; Turaev 2002a; Leidy and Maxim 2006; Friedl and Kim 2005; Friedl and Harvey 2006]. We hope that by determining the indeterminacy of the Alexander polynomials (Theorem 3.1) more information can be extracted from the Alexander polynomials than just the degrees. 
3.4. Proof of Theorem 3.1. We first point out that $\mathbb{K}_{\gamma}\left[t^{ \pm 1}\right]$ is a Euclidean ring with respect to the degree function. This means that given $f(t), g(t) \in \mathbb{K}_{\gamma}\left[t^{ \pm 1}\right] \backslash\{0\}$ we can find $a(t), r(t) \in \mathbb{K}_{\gamma}\left[t^{ \pm 1}\right]$ such that $f(t)=g(t) a(t)+r(t)$ and such that either $r(t)=0$ or $\operatorname{deg} r(t)<\operatorname{deg} g(t)$.

Let $A$ be an $r \times s$ matrix over $\mathbb{K}_{\gamma}\left[t^{ \pm 1}\right]$ of rank $r$. Here and in the following the rank of a matrix over $\mathbb{K}_{\gamma}\left[t^{ \pm 1}\right]$ will be understood as the rank of the matrix considered as a matrix over the skew field $\mathbb{K}_{\gamma}(t)$. Note that $\operatorname{rank}(A)=r$ implies that in particular $s \geq r$. Since $\mathbb{K}_{\gamma}\left[t^{ \pm 1}\right]$ is a Euclidean ring we can perform a sequence of elementary row and column operations to turn $A$ into a matrix of the form $\left(\begin{array}{ll}D & \left.0_{r \times(s-r)}\right)\end{array}\right)$ where $D$ is an $r \times r$ matrix and $0_{r \times(s-r)}$ stands for the $r \times(s-r)$ matrix consisting only of zeros. Since $A$ is of rank $r$ it follows that $D$ has rank $r$ as well, in particular $D$ is a square matrix which is invertible over $\mathbb{K}_{\gamma}(t)$ and we can consider its Dieudonné determinant $\operatorname{det} D$. We define $\operatorname{det} A=\operatorname{det} D \in \mathbb{K}_{\gamma}(t)_{a b}^{\times}$.

Lemma 3.3. Let $A$ be a (square) matrix over $\mathbb{K}_{\gamma}\left[t^{ \pm 1}\right]$ which is invertible over $\mathbb{K} \mathbb{K}_{\gamma}(t)$.

(1) The Dieudonné determinant $\operatorname{det} A \in \mathbb{K}_{\gamma}(t)_{a b}^{\times}$can be represented by an element in $\mathbb{K}_{\gamma}\left[t^{ \pm 1}\right] \backslash\{0\}$.

(2) If $A \in \mathrm{GL}\left(\mathbb{K}_{\gamma}\left[t^{ \pm 1}\right], d\right)$, then $\operatorname{det} A \in \mathbb{K}_{\gamma}(t)_{a b}^{\times}$can be represented by an element of the form $k t^{e}, k \in \mathbb{K}^{\times}, e \in \mathbb{Z}$.

(3) The Dieudonné determinant induces a homomorphism

$$
\operatorname{det}: K_{1}\left(\mathbb{K}_{\gamma}(t)\right) \rightarrow \mathbb{K}_{\gamma}(t)_{a b}^{\times}
$$

sending $K_{1}\left(\mathbb{K}_{\gamma}\left[t^{ \pm 1}\right]\right)$ to $\left\{k t^{e} \mid k \in \mathbb{K}^{\times}, e \in \mathbb{Z}\right\} /\left[\mathbb{K}_{\gamma}(t)^{\times}, \mathbb{K}_{\gamma}(t)^{\times}\right] \subset \mathbb{K}_{\gamma}(t)_{a b}^{\times}$.

Proof. The first statement follows from the discussion preceding the lemma. Now let $A \in \mathrm{GL}\left(\mathbb{K}_{\gamma}\left[t^{ \pm 1}\right], r\right)$. Then $\operatorname{det} A$ has degree zero, by Lemma 3.2 applied to $H=\mathbb{K}_{\gamma}\left[t^{ \pm 1}\right]^{r} / A \mathbb{K}_{\gamma}\left[t^{ \pm 1}\right]^{r}$. This proves the second statement. The last statement follows from the second statement and the fact that the Dieudonne determinant induces a homomorphism det $: K_{1}\left(\mathbb{K}_{\gamma}(t)\right) \rightarrow \mathbb{K}_{\gamma}(t)_{a b}^{\times}$.

Proposition 3.4. Let $A$ be an $r \times s$ matrix over $\mathbb{K}_{\gamma}\left[t^{ \pm 1}\right]$ of rank $r$. Then $\operatorname{det} A \in$ $\mathbb{K}_{\gamma}(t)_{a b}^{\times}$is well defined up to multiplication by an element of the form $k t^{e}, k \in$ $\mathbb{K}^{\times}, e \in \mathbb{Z}$. Furthermore $\operatorname{det} A$ is invariant under elementary row and column operations.

Proof. First note that the effect of an elementary row operation on $A$ over $\mathbb{K}_{\gamma}\left[t^{ \pm 1}\right]$ can be described by left multiplication by a matrix $P \in \mathrm{GL}\left(\mathbb{K}_{\gamma}\left[t^{ \pm 1}\right], r\right)$. Similarly an elementary column operation on $A$ over $\mathbb{K}_{\gamma}\left[t^{ \pm 1}\right]$ can be described by right multiplication by an $s \times s$ matrix $Q \in \mathrm{GL}\left(\mathbb{K}_{\gamma}\left[t^{ \pm 1}\right], s\right)$.

Now assume we have $P_{1}, P_{2} \in \mathrm{GL}\left(\mathbb{K}_{\gamma}\left[t^{ \pm 1}\right], r\right)$ and $Q_{1}, Q_{2} \in \mathrm{GL}\left(\mathbb{K}_{\gamma}\left[t^{ \pm 1}\right], s\right)$ such that $P_{i} A Q_{i}=\left(\begin{array}{ll}D_{i} & 0_{r \times(s-r)}\end{array}\right), i=1,2$ where $D_{i}$ is an $r \times r$ matrix. We are 
done once we show that $\operatorname{det} D_{1}=k t^{e} \operatorname{det} D_{2} \in \mathbb{K}_{\gamma}(t)_{a b}^{\times}$for some $k \in \mathbb{K}^{\times}, e \in \mathbb{Z}$. Let $E_{i}=P_{i}^{-1} D_{i}$. Then by Lemma 3.3 we only have to show that $E_{1}=E_{2} \in$ $K_{1}\left(\mathbb{K}_{\gamma}(t)\right) / K_{1}\left(\mathbb{K}_{\gamma}\left[t^{ \pm 1}\right]\right)$.

We have $\left(\begin{array}{ll}E_{1} & 0\end{array}\right) Q_{1}^{-1}=\left(\begin{array}{ll}E_{2} & 0\end{array}\right) Q_{2}^{-1}$. Let $Q:=Q_{2}^{-1} Q_{1} \in \mathrm{GL}\left(\mathbb{K}_{\gamma}\left[t^{ \pm 1}\right], s\right)$, we therefore get the equality $\left(\begin{array}{ll}E_{1} & 0\end{array}\right)=\left(\begin{array}{ll}E_{2} & 0\end{array}\right) Q$. Now write

$$
Q=\left(\begin{array}{ll}
Q_{11} & Q_{12} \\
Q_{21} & Q_{22}
\end{array}\right)
$$

where $Q_{i j}$ is a $n_{i} \times n_{j}$ matrix over $\mathbb{K}_{\gamma}\left[t^{ \pm 1}\right]$ with $n_{1}=r$ and $n_{2}=s-r$. We get the equality

$$
\left(\begin{array}{cc}
E_{1} & 0 \\
Q_{21} & Q_{22}
\end{array}\right)=\left(\begin{array}{cc}
E_{2} & 0 \\
0 & \mathrm{id}_{s-r}
\end{array}\right)\left(\begin{array}{ll}
Q_{11} & Q_{12} \\
Q_{21} & Q_{22}
\end{array}\right)
$$

It follows in particular that $Q_{22}$ is invertible over $\mathbb{K}_{\gamma}(t)$. Furthermore we have

$$
E_{1} \cdot Q_{22}=E_{2} \in K_{1}\left(\mathbb{K}_{\gamma}(t)\right) / K_{1}\left(\mathbb{K}_{\gamma}\left[t^{ \pm 1}\right]\right) .
$$

Note that deg: $K_{1}\left(\mathbb{K}_{\gamma}(t)\right) \rightarrow \mathbb{Z}$ vanishes on $K_{1}\left(\mathbb{K}_{\gamma}\left[t^{ \pm 1}\right]\right)$ by Lemma 3.3. We therefore get deg det $E_{1}+\operatorname{deg} \operatorname{det} Q_{22}=\operatorname{deg} \operatorname{det} E_{2}$, in particular deg det $E_{1} \leq \operatorname{deg} \operatorname{det} E_{2}$. But by symmetry we have $\operatorname{deg} \operatorname{det} E_{2} \leq \operatorname{deg} \operatorname{det} E_{1}$. In particular deg det $Q_{22}=0$. The proposition now follows immediately from Lemma 3.3 since $\operatorname{deg} f(t)=0$ for $f(t) \in \mathbb{K}_{\gamma}\left[t^{ \pm 1}\right] \backslash\{0\}$ if and only if $f(t)=k t^{e}$ for some $k \in \mathbb{K}^{\times}, e \in \mathbb{Z}$.

The last statement is immediate.

Let $H$ be a finitely generated right $\mathbb{K}_{\gamma}\left[t^{ \pm 1}\right]$-module. We say that an $r \times s$ matrix $A$ is a presentation matrix for $H$ if the following sequence is exact:

$$
\mathbb{K} \mathbb{K}_{\gamma}\left[t^{ \pm 1}\right]^{s} \stackrel{A}{\rightarrow} \mathbb{K}_{\gamma}\left[t^{ \pm 1}\right]^{r} \rightarrow H \rightarrow 0 .
$$

We say that $A$ has full rank if the rank of $A$ equals $r$. Note that $A$ has full rank if and only if $H \otimes_{\mathbb{K}_{\gamma}\left[t^{ \pm 1}\right]} \mathbb{K}_{\gamma}(t)=0$.

The following lemma clearly implies Theorem 3.1.

Lemma 3.5. Let $H$ be a finitely generated right $\mathbb{K}_{\gamma}\left[t^{ \pm 1}\right]$-module and let $A_{1}, A_{2}$ be presentation matrices for $H$. Then $A_{1}$ has full rank if and only if $A_{2}$ has full rank. Furthermore if $A_{i}$ has full rank, then

$$
\operatorname{det} A_{1}=\operatorname{det} A_{2} \in \mathbb{K}_{\gamma}(t)_{a b}^{\times} /\left\{k t^{e} \mid k \in \mathbb{K} \backslash\{0\}, e \in \mathbb{Z}\right\} .
$$

Proof. Two presentation matrices for $H$ differ by a sequence of matrix moves of the following forms and their inverses:

(1) Permutation of rows or columns.

(2) Replacement of the matrix $A$ by $\left(\begin{array}{ll}A & 0 \\ 0 & 1\end{array}\right)$. 
(3) Addition of an extra column of zeros to the matrix $A$.

(4) Addition of a right scalar multiple of a column to another column.

(5) Addition of a left scalar multiple of a row to another row.

This result coincides with [Lickorish 1997, Theorem 6.1] in the commutative case, but the proof there carries through in the case of the base ring $\mathbb{K}_{\gamma}\left[t^{ \pm 1}\right]$ as well (compare [Harvey 2005, Lemma 9.2]).

Clearly none of the moves changes the status of being of full rank, and if a representation is of full rank, then it is easy to see that none of the moves changes the determinant.

3.5. Proof of Theorem 1.1. Now let $X$ be a finite connected CW complex of dimension $n$. Let $\alpha: \pi_{1}(X) \rightarrow \operatorname{GL}\left(\mathbb{K}_{\gamma}\left[t^{ \pm 1}\right], d\right)$ be a representation such that $H_{*}^{\alpha}\left(X ; \mathbb{K}_{\gamma}(t)^{d}\right)=0$. (Recall that we denote the induced representation $\pi_{1}(X) \rightarrow$ $\operatorname{GL}\left(\mathbb{K}_{\gamma}(t), d\right)$ by $\alpha$ as well). Furthermore recall that $\mathbb{K}_{\gamma}(t)$ is flat over $\mathbb{K}_{\gamma}\left[t^{ \pm 1}\right]$, in particular $H_{i}\left(X ; \mathbb{K}_{\gamma}(t)^{d}\right)=H_{i}\left(X ; \mathbb{K}_{\gamma}\left[t^{ \pm 1}\right]^{d}\right) \otimes_{\mathbb{K}_{\gamma}\left[t^{ \pm 1}\right]} \mathbb{K}_{\gamma}(t)$. It follows that $H_{i}\left(X ; \mathbb{K}_{\gamma}(t)^{d}\right)=0$ if and only if $H_{i}\left(X ; \mathbb{K}_{\gamma}\left[t^{ \pm 1}\right]^{d}\right)$ is $\mathbb{K}_{\gamma}\left[t^{ \pm 1}\right]$-torsion, which is equivalent to $\Delta_{i}^{\alpha}(t) \neq 0$. This proves the first statement of Theorem 1.1. To conclude the proof of Theorem 1.1 it remains to prove the following claim.

Claim. If $H_{*}^{\alpha}\left(X ; \mathbb{K}_{\gamma}(t)^{d}\right)=0$, then

$$
\operatorname{det} \tau(X, \alpha)=\prod_{i=0}^{n-1} \Delta_{i}^{\alpha}(t)^{(-1)^{i+1}} \in \mathbb{K}_{\gamma}(t)_{a b}^{\times} /\left\{k t^{e} \mid k \in \mathbb{K} \backslash\{0\}, e \in \mathbb{Z}\right\} .
$$

Proof. Let $C_{*}=C_{*}(\tilde{X}) \otimes_{\mathbb{Z}\left[\pi_{1}(X)\right]} \mathbb{K}_{\gamma}\left[t^{ \pm 1}\right]^{d}$. Any $\mathbb{K}_{\gamma}\left[t^{ \pm 1}\right]$-basis for $C_{*}$ also gives a basis for $C_{*} \otimes_{\mathbb{K}_{\gamma}\left[t^{ \pm 1}\right]} \mathbb{K}_{\gamma}(t)$, which we will always denote by the same symbol.

Denote by $\mathscr{C}_{*}$ the $\mathbb{K}_{\gamma}\left[t^{ \pm 1}\right]$-basis of $C_{*}$ as in Section 2.3. Let

$$
\begin{aligned}
r_{i} & :=\operatorname{dim}_{\mathbb{K}(t)}\left(C_{i} \otimes_{\mathbb{K}_{\gamma}\left[t^{ \pm 1}\right]} \mathbb{K}_{\gamma}(t)\right), \\
s_{i} & :=\operatorname{dim}_{\mathbb{K}(t)}\left(\operatorname{Ker}\left\{C_{i} \otimes_{\mathbb{K}_{\gamma}\left[t^{ \pm 1}\right]} \mathbb{K}_{\gamma}(t) \stackrel{\partial_{i}}{\rightarrow} C_{i-1} \otimes_{\mathbb{K}_{\gamma}\left[t^{ \pm 1}\right]} \mathbb{K}_{\gamma}(t)\right\}\right) .
\end{aligned}
$$

Note that $s_{i}+s_{i-1}=r_{i}$ since $H_{*}\left(X ; \mathbb{K}_{\gamma}(t)^{d}\right)=0$. Note also that $\operatorname{Ker}\left\{C_{i} \stackrel{\partial_{i}}{\rightarrow} C_{i-1}\right\}$ is a free direct summand of $C_{i}$ of rank $s_{i}$ since $\mathbb{K}_{\gamma}\left[t^{ \pm 1}\right]$ is a PID. We can therefore pick $\mathbb{K}_{\gamma}\left[t^{ \pm 1}\right]$-bases $\mathscr{C}_{i}^{\prime}=\left\{v_{1}, \ldots, v_{r_{i}}\right\}$ for $C_{i}$ such that $\left\{v_{1}, \ldots, v_{s_{i}}\right\}$ is a basis for the kernel in question. Base changes from $\mathscr{C}_{i}$ to $\mathscr{C}_{i}^{\prime}$ are given by matrices which are invertible over $\mathbb{K}_{\gamma}\left[t^{ \pm 1}\right]$, so $\tau\left(C_{*} \bigotimes_{\mathbb{K}_{\gamma}\left[t^{ \pm 1}\right]} \mathbb{K}_{\gamma}(t),\left\{\mathscr{C}_{i}\right\}\right)=\tau\left(C_{*} \bigotimes_{\mathbb{K}_{\gamma}\left[t^{ \pm 1}\right]} \mathbb{K}_{\gamma}(t),\left\{\mathscr{C}_{i}^{\prime}\right\}\right) \in K_{1}\left(\mathbb{K}_{\gamma}(t)\right) / K_{1}\left(\mathbb{K}_{\gamma}\left[t^{ \pm 1}\right]\right)$.

Let $A_{i}$ be the $r_{i-1} \times r_{i}$ matrix representing $\partial_{i}: C_{i} \rightarrow C_{i-1}$ with respect to the bases $\mathscr{C}_{i}^{\prime}$ and $\mathscr{C}_{i-1}^{\prime}$. Let $\xi_{i}:=\left\{s_{i}+1, \ldots, r_{i}\right\}, i=1, \ldots, n$ and $\xi_{0}:=\varnothing$. Let $A_{i}(\xi)$ as in 
Theorem 2.1. Note that $A_{i}(\xi)$ is an $s_{i-1} \times s_{i-1}$ matrix over $\mathbb{K}_{\gamma}\left[t^{ \pm 1}\right]$. In particular $\xi:=\left(\xi_{0}, \ldots, \xi_{n}\right)$ is a $\tau$-chain. It is easy to see that

$$
A_{i}=\left(\begin{array}{cc}
0_{s_{i-1} \times s_{i}} & A_{i}(\xi) \\
0_{s_{i-2} \times s_{i}} & 0_{s_{i-2} \times s_{i-1}}
\end{array}\right) .
$$

Since $A_{i}$ has rank $s_{i-1}$ it follows that $A_{i}(\xi)$ is invertible over $\mathbb{K}_{\gamma}(t)$. It follows from Theorem 2.1 that

$$
\tau\left(C_{*} \otimes_{\mathbb{K}_{\gamma}\left[t^{ \pm 1}\right]} \mathbb{K}_{\gamma}(t),\left\{\mathscr{G}_{i}^{\prime}\right\}\right)=\prod_{i=1}^{n} A_{i}(\xi)^{(-1)^{i}} \in K_{1}\left(\mathbb{K}_{\gamma}(t)\right) .
$$

We also have short exact sequences

$$
0 \rightarrow \mathbb{K}_{\gamma}\left[t^{ \pm 1}\right]^{s_{i-1}} \stackrel{A_{i}(\xi)}{\longrightarrow} \mathbb{K}_{\gamma}\left[t^{ \pm 1}\right]^{s_{i-1}} \rightarrow H_{i-1}\left(C_{*}\right)=H_{i-1}\left(X ; \mathbb{K}_{\gamma}\left[t^{ \pm 1}\right]^{d}\right) \rightarrow 0 .
$$

In particular $\left(A_{i}(\xi)\right)$ is a presentation matrix for $H_{i-1}\left(X ; \mathbb{K}_{\gamma}\left[t^{ \pm 1}\right]^{d}\right)$. It therefore follows from Lemma 3.5 that $\operatorname{det} A_{i}(\xi)=\Delta_{i-1}^{\alpha}(t)$.

The following corollary now follows from the fact that deg: $\mathbb{K}_{\gamma}(t)^{\times} \rightarrow \mathbb{Z}$ is a homomorphism and from Lemma 3.2.

Corollary 3.6. Let $X$ be a finite connected $C W$ complex of dimension $n$. Let $\alpha: \pi_{1}(X) \rightarrow \operatorname{GL}\left(\mathbb{K}_{\gamma}\left[t^{ \pm 1}\right], d\right)$ be a representation such that $H_{*}^{\alpha}\left(X ; \mathbb{K}_{\gamma}(t)^{d}\right)=0$. Then

$$
\operatorname{deg} \tau(X, \alpha)=\sum_{i=0}^{n-1}(-1)^{i+1} \operatorname{deg} \Delta_{i}^{\alpha}(t)=\sum_{i=0}^{n-1}(-1)^{i+1} \operatorname{dim}_{\mathbb{K}}\left(H_{i}\left(X ; \mathbb{K}_{\gamma}\left[t^{ \pm 1}\right]^{d}\right) .\right.
$$

Remark. In the case that $H_{*}\left(X ; \mathbb{K}_{\gamma}(t)^{d}\right) \neq 0$ we can pick $\mathbb{K}_{\gamma}\left[t^{ \pm 1}\right]$-bases $\mathscr{H}_{i}$ for the $\mathbb{K}_{\gamma}\left[t^{ \pm 1}\right]$-free parts of $H_{i}\left(X ; \mathbb{K}_{\gamma}\left[t^{ \pm 1}\right]^{d}\right)$. These give bases for $H_{i}\left(X ; \mathbb{K}_{\gamma}(t)^{d}\right)=$ $H_{i}\left(X ; \mathbb{K}_{\gamma}\left[t^{ \pm 1}\right]^{d}\right) \otimes_{\mathbb{K}_{\gamma}\left[t^{ \pm 1}\right]} \mathbb{K}_{\gamma}(t)$ and we can consider

$$
\tau\left(X, \alpha,\left\{\mathscr{H}_{i}\right\}\right)=\tau\left(C_{*}(\tilde{X}) \otimes_{\mathbb{Z}\left[\pi_{1}(X)\right]} \mathbb{K}_{\gamma}(t)^{d},\left\{\mathscr{H}_{i}\right\}\right) \in K_{1}\left(\mathbb{K}_{\gamma}(t)\right)
$$

(see [Milnor 1966] for details). As an element of $K_{1}\left(\mathbb{K}_{\gamma}(t)\right) / K_{1}\left(\mathbb{K}_{\gamma}\left[t^{ \pm 1}\right]\right)$, this $\tau\left(X, \alpha,\left\{\mathscr{H}_{i}\right\}\right)$ is independent of the choice of $\left\{\mathscr{H}_{i}\right\}$. The proof of Theorem 1.1 can be generalized to show that it is the alternating product of the orders of the $\mathbb{K}_{\gamma}\left[t^{ \pm 1}\right.$ ]-torsion submodules of $H_{*}\left(X ; \mathbb{K}_{\gamma}\left[t^{ \pm 1}\right]^{d}\right)$ (compare [Kirk and Livingston 1999] in the commutative case).

\section{3-manifolds and 2-complexes}

We now restrict ourselves to $\phi$-compatible representations since these have a closer connection to the topology of a space. 
Lemma 4.1. Let $X$ be a connected $C W$ complex with finitely many cells in dimensions 0 and 1 . Let $\phi \in H^{1}(X ; \mathbb{Z})$ nontrivial and let $\alpha: \pi_{1}(X) \rightarrow \mathrm{GL}\left(\mathbb{K}_{\gamma}\left[t^{ \pm 1}\right], d\right)$ be a $\phi$-compatible representation. Then $\Delta_{0}^{\alpha}(t) \neq 0$. If $X$ is in fact an $k$-manifold, then $\Delta_{k}^{\alpha}(t)=1$.

We need the following notation. If $A=\left(a_{i j}\right)$ is an $r \times s$ matrix over $\mathbb{Z}\left[\pi_{1}(X)\right]$ and $\alpha: \pi_{1}(X) \rightarrow \operatorname{GL}(R, d)$ a representation. Then we denote by $\alpha(A)$ the $r d \times s d$ matrix over $R$ obtained by replacing each entry $a_{i j} \in \mathbb{Z}\left[\pi_{1}(X)\right]$ of $A$ by the $d \times d$ matrix $\alpha\left(a_{i j}\right)$.

Proof. First equip $X$ with a CW structure with one 0 -cell and $n 1$-cells $g_{1}, \ldots, g_{n}$. We denote the corresponding elements in $\pi_{1}(X)$ by $g_{1}, \ldots, g_{n}$ as well. Since $\phi$ is nontrivial there exists at least one $i$ such that $\phi\left(g_{i}\right) \neq 0$. Write $C_{*}=C_{*}(\tilde{X}) \otimes_{\mathbb{Z}\left[\pi_{1}(X)\right]}$ $\mathbb{K}_{\gamma}\left[t^{ \pm 1}\right]^{d}$. The boundary map $\partial_{1}: C_{1} \rightarrow C_{0}$ is represented by the matrix

$$
\left(\alpha\left(1-g_{1}\right), \ldots, \alpha\left(1-g_{n}\right)\right)=\left(\mathrm{id}-\alpha\left(g_{1}\right), \ldots, \mathrm{id}-\alpha\left(g_{n}\right)\right) .
$$

Since $\alpha$ is $\phi$-compatible it follows that $\alpha\left(1-g_{i}\right)=\mathrm{id}-A t^{\phi\left(g_{i}\right)}$ for some matrix $A \in \mathrm{GL}(\mathbb{K}, d)$. The first statement of the lemma now follows from Lemma 4.2.

If $X$ is a closed $k$-manifold then equip $X$ with a CW structure with one $k$-cell. Since $\phi$ is primitive and $\phi$-compatible an argument as above shows that $\partial_{k}: C_{k} \rightarrow$ $C_{k-1}$ has full rank, i.e. $H_{k}\left(X ; \mathbb{K}_{\gamma}\left[t^{ \pm 1}\right]^{d}\right)=0$. Hence $\Delta_{k}^{\alpha}(t)=1$. If $X$ is a $k$ manifold with boundary, then it is homotopy equivalent to a $(k-1)$-complex, and hence $H_{k}\left(X ; \mathbb{K}_{\gamma}\left[t^{ \pm 1}\right]^{d}\right)=0$.

Lemma 4.2. Let $\mathbb{K}_{\gamma}\left[t^{ \pm 1}\right]$ be a skew Laurent polynomial ring and let $A, B$ be invertible $d \times d$ matrices over $\mathbb{K}$ and $r \neq 0$. Then $\operatorname{deg} \operatorname{det}\left(A+B t^{r}\right)=d r$. In particular $A+B t^{r}$ is invertible over $\mathbb{K}_{\gamma}(t)$.

Harvey has proved a related result [2005, Proposition 9.1].

Proof. We can clearly assume that $r>0$. Let $\left\{e_{1}, \ldots, e_{d}\right\}$ be a basis for $\mathbb{K}^{d}$. Consider the projection map $p: \mathbb{K}_{\gamma}\left[t^{ \pm 1}\right]^{d} \rightarrow P=\mathbb{K}_{\gamma}\left[t^{ \pm 1}\right]^{d} /\left(A+B t^{r}\right) \mathbb{K}_{\gamma}\left[t^{ \pm 1}\right]^{d}$. By Lemma 3.2 we are done once we show that $p\left(e_{i} t^{j}\right), i \in\{1, \ldots, d\}, j \in\{0, \ldots, r-$ 1 \} form a basis for $P$ as a right $\mathbb{K}$-vector space.

It follows easily from the fact that $A, B$ are invertible that this is indeed a generating set. Let $v \in \mathbb{K}_{\gamma}\left[t^{ \pm 1}\right]^{d} \backslash\{0\}$. We can write $v=\sum_{i=n}^{m} v_{i} t^{i}, v_{i} \in \mathbb{K}^{d}$ with $v_{n} \neq 0, v_{m} \neq 0$. Since $A, B$ are invertible it follows that $\left(A+B t^{r}\right) v$ has terms with $t$-exponent $n$ and terms with $t$-exponent $m+r$. This observation can be used to show that the vectors in question are linearly independent in $P$.

We can now give the proof of Theorem 1.2.

Proof of Theorem 1.2. Now let $M$ be a 3-manifold whose boundary is empty or consists of tori. A standard duality argument shows that $2 \chi(M)=\chi(\partial M)=0$. 
Let $\phi \in H^{1}(M ; \mathbb{Z})$ be nontrivial, and $\alpha: \pi_{1}(M) \rightarrow \mathrm{GL}\left(\mathbb{K}_{\gamma}\left[t^{ \pm 1}\right], d\right)$ a $\phi$-compatible representation.

We first show that $H_{*}\left(M ; \mathbb{K}_{\gamma}(t)^{d}\right)=0$ if and only if $\Delta_{1}^{\alpha}(t) \neq 0$. In Section we showed that $H_{i}\left(M ; \mathbb{K}_{\gamma}(t)^{d}\right)=0$ if and only if $\Delta_{i}^{\alpha}(t) \neq 0$. It now follows from Lemma 4.1 that $H_{i}\left(M ; \mathbb{K}_{\gamma}(t)^{d}\right)=0$ for $i=0$, 3. If $\Delta_{1}^{\alpha}(t) \neq 0$, then $H_{1}\left(M ; \mathbb{K}_{\gamma}(t)^{d}\right)=0$. Since $\chi\left(H_{i}\left(M ; \mathbb{K}_{\gamma}(t)^{d}\right)\right)=d \chi(M)=0$ it follows that $H_{2}\left(M ; \mathbb{K}_{\gamma}(t)^{d}\right)=0$.

Claim. $\|\phi\|_{T}$ is bounded below by

$\frac{1}{d}\left(-\operatorname{dim}_{\mathbb{K}} H_{0}^{\alpha}\left(M ; \mathbb{K}_{\gamma}\left[t^{ \pm 1}\right]^{d}\right)+\operatorname{dim}_{\mathbb{}} H_{1}^{\alpha}\left(M ; \mathbb{K}_{\gamma}\left[t^{ \pm 1}\right]^{d}\right)-\operatorname{dim}_{\mathbb{K}} H_{2}^{\alpha}\left(M ; \mathbb{K}_{\gamma}\left[t^{ \pm 1}\right]^{d}\right)\right)$.

This inequality becomes an equality if $(M, \phi)$ fibers over $S^{1}$ and if $M \neq S^{1} \times D^{2}$, $M \neq S^{1} \times S^{2}$.

Proof. If $\phi$ vanishes on $X \subset M$, the restriction of $\alpha$ to $\pi_{1}(X)$ lies in $\operatorname{GL}(\mathbb{K}, d) \subset$ $\operatorname{GL}\left(\mathbb{K}_{\gamma}\left[t^{ \pm 1}\right], d\right)$ since $\alpha$ is $\phi$-compatible. Therefore

$$
H_{i}^{\alpha}\left(X ; \mathbb{K}_{\gamma}\left[t^{ \pm 1}\right]^{d}\right) \cong H_{i}^{\alpha}\left(X ; \mathbb{K}^{d}\right) \mathbb{\mathbb { K }}_{\mathbb{K}} \mathbb{K}_{\gamma}\left[t^{ \pm 1}\right] .
$$

The proofs of [Friedl and Kim 2006, Theorem 3.1 and Theorem 6.1] can now easily be translated to this noncommutative setting. This proves the claim.

Combining the results of the claim with Lemma 3.2 and Corollary 3.6 we immediately get a proof for Theorem 1.2.

In order to relate Theorem 1.2 to the results of [Cochran 2004; Harvey 2005; Turaev 2002a] we need the following computations for one-dimensional $\phi$-compatible representations. Recall that $\phi \in H^{1}(X ; \mathbb{Z})$ is called primitive if the corresponding $\operatorname{map} \phi: H_{1}(X ; \mathbb{Z}) \rightarrow \mathbb{Z}$ is surjective.

Lemma 4.3. Let $X$ be a connected $C W$ complex with finitely many cells in dimensions zero and one. Let $\phi \in H^{1}(X ; \mathbb{Z})$ primitive. Let $\alpha: \pi_{1}(X) \rightarrow \operatorname{GL}\left(\mathbb{K}_{\gamma}\left[t^{ \pm 1}\right], 1\right)$ be a $\phi$-compatible one-dimensional representation. If $\alpha\left(\pi_{1}(X)\right) \subset \mathrm{GL}\left(\mathbb{K}_{\gamma}\left[t^{ \pm 1}\right], 1\right)$ is cyclic, then $\Delta_{0}^{\alpha}(t)=a t-1$ for some $a \in \mathbb{K} \backslash\{0\}$. Otherwise $\Delta_{0}^{\alpha}(t)=1$.

Proof. Equip $X$ with a CW structure with one 0-cell and then consider the chain complex for $X$ as in Lemma 4.1. The lemma now follows easily from the observation that in $\mathbb{K}_{\gamma}\left[t^{ \pm 1}\right]$ we have $\operatorname{gcd}(1-a t, 1-b t)=1$ if $a \neq b \in \mathbb{K}$.

Lemma 4.4. Let $X$ be a 3-manifold with empty or toroidal boundary or let $X$ be a 2-complex with $\chi(X)=0$. Let $\phi \in H^{1}(M ; \mathbb{Z})$ nontrivial. Let $\alpha: \pi_{1}(M) \rightarrow$ $\mathrm{GL}\left(\mathbb{K}_{\gamma}\left[t^{ \pm 1}\right], 1\right)$ be a $\phi$-compatible one-dimensional representation. Assume that $\Delta_{1}^{\alpha}(t) \neq 0$. If $X$ is a closed 3-manifold, then $\operatorname{deg} \Delta_{2}^{\alpha}(t)=\operatorname{deg} \Delta_{0}^{\alpha}(t)$, otherwise $\Delta_{2}^{\alpha}(t)=1$. 
Proof. First assume that $X$ is a 3-manifold. Then the lemma follows from combining [Turaev 2002a, Sections 4.3 and 4.4] with [Friedl and Kim 2006, Lemmas 4.7 and 4.9]. (The latter results also hold in the noncommutative setting.) If $X$ is a 2-complex then the argument in the proof of Theorem 1.2 shows that $H_{2}\left(X ; \mathbb{K}_{\gamma}(t)^{d}\right)=0$. But since $X$ is a 2-complex we have $H_{2}\left(X ; \mathbb{K}_{\gamma}\left[t^{ \pm 1}\right]^{d}\right) \subset$ $H_{2}\left(X ; \mathbb{K}_{\gamma}(t)^{d}\right)$, hence $H_{2}\left(X ; \mathbb{K}_{\gamma}\left[t^{ \pm 1}\right]^{d}\right)=0$ and $\Delta_{2}^{\alpha}(t)=1$.

Remark. We cannot apply the duality results of [Friedl and Kim 2006, Lemma 4.12 and Proposition 4.13] since the natural involution on $\mathbb{Z}[G]$ does not necessarily extend to an involution on $\mathbb{K}_{\gamma}\left[t^{ \pm 1}\right]$, i.e., the representation $\mathbb{Z}[G] \rightarrow \mathbb{K}_{\gamma}\left[t^{ \pm 1}\right]$ is not necessarily unitary.

It now follows immediately from Lemma 4.3 and 4.4 and the discussion in Section 5 that Theorem 1.2 contains the results of [McMullen 2002; Cochran 2004; Harvey 2005; Turaev 2002a; Friedl and Kim 2006].

Remark. Given a 2-complex $X$, Turaev [2002b] defined a norm

$$
\|\|_{X}: H^{1}(X ; \mathbb{R}) \rightarrow \mathbb{R},
$$

modeled on the definition of the Thurston norm of a 3-manifold. He then gave lower bounds for the Turaev norm (see also [Turaev 2002a]) which have the same form as certain lower bounds for the Thurston norm. Going through the proofs in [Friedl and Kim 2006] it is not hard to see that the obvious version of Theorem 1.2 for 2-complexes also holds.

If $M$ is a 3-manifold with boundary, then it is homotopy equivalent to a 2complex $X$. It is not known whether the Thurston norm of $M$ agrees with the Turaev norm on $X$. But the fact that Theorem 1.2 holds in both cases, and the observation that $\operatorname{deg} \tau(X, \alpha)$ is a homotopy invariant by Theorem 1.1 suggests that they do in fact agree.

\section{Examples for skew fields and $\phi$-compatible representations}

5.1. Skew fields of group rings. A group $G$ is called locally indicable if for every finitely generated subgroup $U \subset G$ there exists a nontrivial homomorphism $U \rightarrow \mathbb{Z}$.

Theorem 5.1. Let $G$ be a locally indicable and amenable group and let $\mathbb{F}$ be a commutative field. Then the following hold.

(1) $\mathbb{F}[G]$ is an Ore domain, in particular it embeds in its classical right ring of quotients $\mathbb{K}(G)$.

(2) $\mathbb{K}(G)$ is flat over $\mathbb{F}[G]$.

It follows from [Higman 1940] that $\mathbb{F}[G]$ has no zero divisors. The first part now follows from [Tamari 1957] or [Dodziuk et al. 2003, Corollary 6.3]. Part (b) 
is a property of Ore localizations (see [Ranicki 1998, p. 99], for example). We call $\mathbb{K}(G)$ the Ore localization of $\mathbb{F}[G]$.

A group $G$ is called poly-torsion-free-abelian (PTFA) if there exists a filtration

$$
1=G_{0} \subset G_{1} \subset \cdots \subset G_{n-1} \subset G_{n}=G
$$

such that $G_{i} / G_{i-1}$ is torsion free abelian. PTFA groups are amenable and locally indicable [Strebel 1974]. The group rings of PTFA groups played an important role in [Cochran et al. 2003; Cochran 2004; Harvey 2005].

5.2. Examples for $\phi$-compatible representations. Let $X$ be a connected $\mathrm{CW}$ complex and $\phi \in H^{1}(X ; \mathbb{Z})$. We give examples of $\phi$-compatible representations.

Let $\mathbb{F}$ be a commutative field. The element $\phi \in H^{1}(X ; \mathbb{Z}) \cong \operatorname{Hom}\left(H_{1}(X ; \mathbb{Z}),\langle t\rangle\right)$ induces a $\phi$-compatible representation $\phi: \mathbb{Z}\left[\pi_{1}(X)\right] \rightarrow \mathbb{F}\left[t^{ \pm 1}\right]$. Furthermore if $\alpha: \pi_{1}(X) \rightarrow \operatorname{GL}(\mathbb{F}, d)$ is a representation, then $\pi_{1}(X)$ acts via $\alpha \otimes \phi$ on the $\mathbb{F}\left[t^{ \pm 1}\right]-$ module $\mathbb{F}^{d} \otimes_{\mathbb{F}} \mathbb{F}\left[t^{ \pm 1}\right] \cong \mathbb{F}\left[t^{ \pm 1}\right]^{d}$. We therefore get a representation $\alpha \otimes \phi: \pi_{1}(X) \rightarrow$ $\mathrm{GL}\left(\mathbb{F}\left[t^{ \pm 1}\right], d\right)$, which is clearly $\phi$-compatible. In this particular case Theorem 1.2 was proved in [Friedl and Kim 2006].

To describe the $\phi$-compatible representations of Cochran [2004] and Harvey [2005; 2006] we need the following definition.

Definition. Let $\pi$ be a group, $\phi: \pi \rightarrow \mathbb{Z}$ an epimorphism and $\varphi: \pi \rightarrow G$ an epimorphism to a locally indicable and amenable group $G$ such that there exists a map $\phi_{G}: G \rightarrow \mathbb{Z}$ (which is necessarily unique) such that

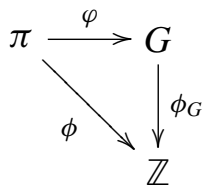

commutes. Following [Harvey 2006, Definition 1.4] we call $(\varphi, \phi)$ an admissible pair. If $\phi_{G}$ is an isomorphism, then $(\varphi, \phi)$ is called initial.

Now let $\left(\varphi: \pi_{1}(X) \rightarrow G, \phi\right)$ be an admissible pair for $\pi_{1}(X)$. In the following we denote $\operatorname{Ker}\{\phi: G \rightarrow \mathbb{Z}\}$ by $G^{\prime}(\phi)$. When the homomorphism $\phi$ is understood we will write $G^{\prime}$ for $G^{\prime}(\phi)$. Clearly $G^{\prime}$ is still a locally indicable and amenable group. Let $\mathbb{F}$ be any commutative field and $\mathbb{K}\left(G^{\prime}\right)$ the Ore localization of $\mathbb{F}[G]$. Pick an element $\mu \in G$ such that $\phi(\mu)=1$. Let $\gamma: \mathbb{K}\left(G^{\prime}\right) \rightarrow \mathbb{K}\left(G^{\prime}\right)$ be the homomorphism given by $\gamma(a)=\mu a \mu^{-1}$. Then we get a representation

$$
\begin{aligned}
G & \rightarrow \mathrm{GL}\left(\mathbb{K}\left(G^{\prime}\right)_{\gamma}\left[t^{ \pm 1}\right], 1\right) \\
g & \mapsto\left(g \mu^{-\phi(g)} t^{\phi(g)}\right) .
\end{aligned}
$$

It is clear that $\alpha: \pi_{1}(X) \rightarrow G \rightarrow \operatorname{GL}\left(\mathbb{K}\left(G^{\prime}\right)_{\gamma}\left[t^{ \pm 1}\right], 1\right)$ is $\phi$-compatible. Note that the ring $\mathbb{K}\left(G^{\prime}\right)_{\gamma}\left[t^{ \pm 1}\right]$, and hence the representation above, depend on the 
choice of $\mu$. We will nonetheless suppress $\gamma$ from the notation since different choices of splittings give isomorphic rings. We often make use of the fact that $f(t) g(t)^{-1} \rightarrow f(\mu) g(\mu)^{-1}$ defines an isomorphism $\mathbb{K}\left(G^{\prime}\right)(t) \rightarrow \mathbb{K}(G)$ (see [Harvey 2005 , Proposition 4.5]). Similarly $\mathbb{Z}\left[G^{\prime}\right]\left[t^{ \pm 1}\right] \stackrel{\cong}{\rightarrow}[G]$.

An important example of admissible pairs is provided by Harvey's rational derived series of a group $G$; see [Harvey 2005, Section 3]. Let $G_{r}^{(0)}=G$ and define inductively

$$
G_{r}^{(n)}=\left\{g \in G_{r}^{(n-1)} \mid g^{k} \in\left[G_{r}^{(n-1)}, G_{r}^{(n-1)}\right] \text { for some } k \in \mathbb{Z} \backslash\{0\}\right\} .
$$

Note that $G_{r}^{(n-1)} / G_{r}^{(n)} \cong\left(G_{r}^{(n-1)} /\left[G_{r}^{(n-1)}, G_{r}^{(n-1)}\right]\right) / \mathbb{Z}$-torsion. By [Harvey 2005, Corollary 3.6] the quotients $G / G_{r}^{(n)}$ are PTFA groups for any $G$ and any $n$. If $\phi: G \rightarrow \mathbb{Z}$ is an epimorphism, then $\left(G \rightarrow G / G_{r}^{(n)}, \phi\right)$ is an admissible pair for $(G, \phi)$ for any $n>0$.

For example, if $K$ is a knot and $G=\pi_{1}\left(S^{3} \backslash K\right)$, it follows from [Strebel 1974] that $G_{r}^{(n)}=G^{(n)}$, i.e. the rational derived series equals the ordinary derived series (compare [Cochran 2004; Harvey 2005]).

Remark. Recall that for a knot $K$ the knot exterior $S^{3} \backslash v K$ is denoted by $X(K)$. Let $\pi=\pi_{1}(X(K))$ and let $\phi \in H^{1}(X(K) ; \mathbb{Z})$ primitive. Then

$$
\bar{\delta}_{n}(K)=\operatorname{dim}_{\mathbb{K}\left(\pi^{\prime} /\left(\pi^{\prime}\right)_{r}^{(n)}\right)}\left(H_{1}\left(X(K), \mathbb{K}\left(\pi^{\prime} /\left(\pi^{\prime}\right)_{r}^{(n)}\right)\left[t^{ \pm 1}\right]\right)\right.
$$

is a knot invariant for $n>0$. Cochran [2004, p. 395, Question 5] asked whether $K \mapsto \bar{\delta}_{n}(K)$ is of finite type.

Eisermann [2000, Lemma 7] has shown that the genus is not a finite type knot invariant. Cochran [2004] showed that $\bar{\delta}_{n}(K) \leq 2$ genus $(K)$ (see also Theorem 1.2 together with Corollary 3.6 and Lemmas 4.3 and 4.4). Eisermann's argument can now be used to show that $K \mapsto \bar{\delta}_{n}(K)$ is not of finite type either.

Let $X$ be again be a connected CW complex and $\phi \in H^{1}(X ; \mathbb{Z})$. The two types of $\phi$-compatible representations given above can be combined as follows. Let $\alpha$ : $\pi_{1}(X) \rightarrow \mathrm{GL}(\mathbb{F}, d)$ be a representation and let $\varphi: \pi_{1}(X) \rightarrow G$ be a homomorphism such that $(\varphi, \phi)$ is an admissible pair. Denote the Ore localization of $\mathbb{F}\left[G^{\prime}\right]$ by $\mathbb{K}\left(G^{\prime}\right)$. Then $\pi_{1}(X)$ acts via $\varphi \otimes \alpha$ on $\mathbb{K}\left(G^{\prime}\right)\left[t^{ \pm 1}\right] \otimes_{\mathbb{F}} \mathbb{F}^{d} \cong \mathbb{K} \ll\left(G^{\prime}\right)\left[t^{ \pm 1}\right]^{d}$. We therefore get a $\phi$-compatible representation $\varphi \otimes \alpha: \pi_{1}(X) \rightarrow \operatorname{GL}\left(\mathbb{K}\left(G^{\prime}\right)\left[t^{ \pm 1}\right], d\right)$.

\section{Comparing different $\phi$-compatible maps}

Definition [Harvey 2006]. Let $\pi$ be a group and $\phi: \pi \rightarrow \mathbb{Z}$ an epimorphism. Furthermore let $\varphi_{1}: \pi \rightarrow G_{1}$ and $\varphi_{2}: \pi \rightarrow G_{2}$ be epimorphisms to locally indicable and amenable groups $G_{1}$ and $G_{2}$. We call $\left(\varphi_{1}, \varphi_{2}, \phi\right)$ an admissible triple for $\pi$ 
if there exist epimorphisms $\varphi_{2}^{1}: G_{1} \rightarrow G_{2}$ (which is not an isomorphism) and $\phi_{2}: G_{2} \rightarrow \mathbb{Z}$ such that $\varphi_{2}=\varphi_{2}^{1} \circ \varphi_{1}$, and $\phi=\phi_{2} \circ \varphi_{2}$.

The situation can be summarized in the diagram

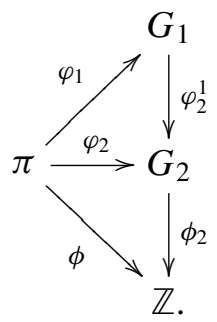

In particular, $\left(\varphi_{i}, \phi\right), i=1,2$, are admissible pairs for $\pi$. Given an admissible triple we can pick splittings $\mathbb{Z} \rightarrow G_{i}$ of $\varphi_{i}, i=1,2$ which make the following diagram commute:

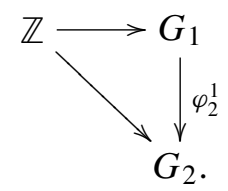

We therefore get an induced commutative diagram of ring homomorphisms

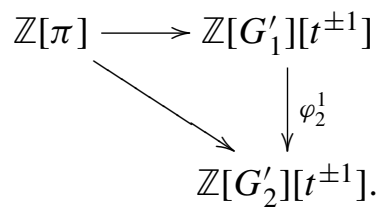

(We are suppressing the notation for the twisting in the skew Laurent polynomial rings.) Denote the $\phi$-compatible maps $\mathbb{Z}[\pi] \rightarrow \mathbb{K}\left(G_{i}^{\prime}\right)\left[t^{ \pm 1}\right], i=1,2$ by $\varphi_{i}$ as well. For convenience we recall Theorem 1.3.

Theorem 1.3. Let $M$ be a 3-manifold whose boundary is a (possibly empty) collection of tori or let $M$ be a 2-complex with $\chi(M)=0$. Let $\alpha: \pi_{1}(M) \rightarrow \mathrm{GL}(\mathbb{F}, d)$ be a representation and $\left(\varphi_{1}, \varphi_{2}, \phi\right)$ an admissible triple for $\pi_{1}(M)$. If $\tau\left(M, \varphi_{2} \otimes \alpha\right) \neq 0$, then $\tau\left(M, \varphi_{1} \otimes \alpha\right) \neq 0$. Furthermore in that case

$$
\operatorname{deg} \tau\left(M, \varphi_{1} \otimes \alpha\right) \geq \operatorname{deg} \tau\left(M, \varphi_{2} \otimes \alpha\right) .
$$

6.1. Proof of Theorem 1.3 for closed 3-manifolds. Let $M$ be a closed 3-manifold. Choose a triangulation of $M$. Let $T$ be a maximal tree in the 1-skeleton of the triangulation and let $T^{\prime}$ be a maximal tree in the dual 1-skeleton. Following [McMullen 2002, Section 5] we collapse $T$ to form a single 0-cell and join the 3-simplices along $T^{\prime}$ to form a single 3-cell. Since $\chi(M)=0$ the number $n$ of 1-cells equals 
the number of 2-cells. Consider the chain complex of the universal cover $\tilde{M}$ :

$$
0 \rightarrow C_{3}(\tilde{M})^{1} \stackrel{\partial_{3}}{\rightarrow} C_{2}(\tilde{M})^{n} \stackrel{\partial_{2}}{\rightarrow} C_{1}(\tilde{M})^{n} \stackrel{\partial_{1}}{\rightarrow} C_{0}(\tilde{M})^{1} \rightarrow 0,
$$

where the superscript indicates the rank over $\mathbb{Z}\left[\pi_{1}(M)\right]$. Picking appropriate lifts of the (oriented) cells of $M$ to cells of $\tilde{M}$ we get bases $\tilde{\sigma}_{i}=\left\{\tilde{\sigma}_{i}^{1}, \ldots, \tilde{\sigma}_{i}^{r_{i}}\right\}$ for the $\mathbb{Z}\left[\pi_{1}(M)\right]$-modules $C_{i}(\tilde{M})$, such that if $A_{i}$ denotes the matrix corresponding to $\partial_{i}$, then $A_{1}$ and $A_{3}$ are of the form

$$
\begin{aligned}
& A_{3}=\left(1-g_{1}, \ldots, 1-g_{n}\right)^{t}, \quad g_{i} \in \pi_{1}(M) \\
& A_{1}=\left(1-h_{1}, \ldots, 1-h_{n}\right), \quad h_{i} \in \pi_{1}(M) .
\end{aligned}
$$

Clearly $\left\{h_{1}, \ldots, h_{n}\right\}$ is a generating set for $\pi_{1}(M)$. Since $M$ is a closed 3-manifold $\left\{g_{1}, \ldots, g_{n}\right\}$ is a generating set for $\pi_{1}(M)$ as well. In particular we can find $k, l \in$ $\{1, \ldots, n\}$ such that $\phi\left(g_{k}\right) \neq 0, \phi\left(h_{l}\right) \neq 0$.

In the following we write $\alpha_{i}=\varphi_{i} \otimes \alpha: \pi_{1}(M) \rightarrow \operatorname{GL}\left(\mathbb{K}\left(G_{i}^{\prime}\right)\left[t^{ \pm 1}\right] \otimes \mathbb{F}^{d}\right) \rightarrow$ $\operatorname{GL}\left(\mathbb{K}\left(G_{i}^{\prime}\right)\left[t^{ \pm 1}\right], d\right), i=1,2$ and we write $\varphi=\varphi_{2}^{1}$.

Lemma 6.1. We have

$$
\begin{aligned}
& \operatorname{deg}\left(\alpha_{1}\left(1-h_{l}\right)\right)=\operatorname{deg}\left(\alpha_{2}\left(1-h_{l}\right)\right)=d\left|\phi\left(h_{l}\right)\right|, \\
& \operatorname{deg}\left(\alpha_{1}\left(1-g_{k}\right)\right)=\operatorname{deg}\left(\alpha_{2}\left(1-g_{k}\right)\right)=d\left|\phi\left(g_{k}\right)\right| .
\end{aligned}
$$

In particular, the matrices $\alpha_{i}\left(1-h_{l}\right), \alpha_{i}\left(1-g_{k}\right)$ are invertible over $\mathbb{K}\left(G_{i}^{\prime}\right)(t)$ for $i=1,2$.

Proof. Note that $\alpha_{i}\left(1-h_{l}\right)=\mathrm{id}-\alpha_{i}\left(h_{l}\right), \alpha_{i}\left(1-g_{k}\right)=\mathrm{id}-\alpha_{i}\left(g_{k}\right)$ and that $\phi\left(h_{l}\right) \neq 0, \phi\left(g_{k}\right) \neq 0$. The lemma now follows from Lemma 4.2 since $\alpha_{1}$ and $\alpha_{2}$ are $\phi$-compatible.

Denote by $B$ the result of deleting the $k$-column and the $l$-row of $A_{2}$.

Lemma 6.2. $\tau\left(M, \alpha_{i}\right) \neq 0$ if and only if $\alpha_{i}(B)$ is invertible. Furthermore if $\tau\left(M, \alpha_{i}\right) \neq 0$, then

$$
\tau\left(M, \alpha_{i}\right)=\alpha_{i}\left(1-g_{k}\right)^{-1} \alpha_{i}(B) \alpha_{i}\left(1-h_{l}\right)^{-1} \in K_{1}\left(\mathbb{K}\left(G_{i}^{\prime}\right)(t)\right) / \pm \alpha_{i}\left(\pi_{1}(M)\right) .
$$

Proof. Denote the standard basis of $\mathbb{K}\left(G_{i}^{\prime}\right)(t)^{d}$ by $e_{1}, \ldots, e_{d}$. We equip $C_{j}=$ $C_{j}^{\alpha_{i}}\left(M ; \mathbb{K}\left(G_{i}^{\prime}\right)(t)^{d}\right)$ with the ordered bases

$$
\mathscr{C}_{j}=\left\{\tilde{\sigma}_{j}^{1} \otimes e_{1}, \ldots, \tilde{\sigma}_{j}^{1} \otimes e_{d}, \ldots, \tilde{\sigma}_{i}^{r_{j}} \otimes e_{1}, \ldots, \tilde{\sigma}_{i}^{r_{j}} \otimes e_{d}\right\} .
$$

Now let

$$
\begin{aligned}
& \xi_{0}=\varnothing, \\
& \xi_{1}=\{l d+1, \ldots, l(d+1)\}, \\
& \xi_{2}=\{1, \ldots, n d\} \backslash\{k d+1, \ldots,(k+1) d\}, \\
& \xi_{3}=\{1, \ldots, d\} .
\end{aligned}
$$


Then $\xi=\left(\xi_{0}, \xi_{1}, \xi_{2}, \xi_{3}\right)$ is a $\tau$-chain for $C_{*}$. We have $A_{1}(\xi)=\alpha_{i}\left(1-h_{l}\right), A_{2}(\xi)=$ $\alpha_{i}(B)$ and $A_{3}(\xi)=\alpha_{i}\left(1-g_{k}\right)$. Clearly $A_{1}(\xi)$ and $A_{3}(\xi)$ are invertible by Lemma 6.1. The proposition now follows immediately from Theorem 2.1.

Now assume that $\tau\left(M, \alpha_{2}\right) \neq 0$. Then $\alpha_{2}(B)$ is invertible over $\mathbb{K}\left(G_{2}^{\prime}\right)(t)$ by Lemma 6.2. Note that $\alpha_{i}(B)$ is defined over $\mathbb{Z}\left[G_{i}^{\prime}\right]\left[t^{ \pm 1}\right] \subset \mathbb{K}\left(G_{i}^{\prime}\right)(t)$. In particular $\alpha_{2}(B)=\varphi\left(\alpha_{1}(B)\right)$. It follows from the following lemma that $\alpha_{1}(B)$ is invertible as well.

Lemma 6.3. Let $P$ be an $r \times s$ matrix over $\mathbb{Z}\left[G_{1}^{\prime}\right]\left[t^{ \pm 1}\right]$. If

$$
\begin{aligned}
\mathbb{Z}\left[G_{2}\right]^{s} & \rightarrow \mathbb{Z}\left[G_{2}\right]^{r} \\
v & \mapsto \varphi(P) v
\end{aligned}
$$

is invertible over $\mathbb{K}\left(G_{2}^{\prime}\right)(t)$, then $P$ is invertible over $\mathbb{K}\left(G_{1}^{\prime}\right)(t)$. The same holds with "invertible" replaced by "injective".

Proof. Assume that multiplication by $\varphi(P)$ is injective over $\mathbb{K}\left(G_{2}^{\prime}\right)(t)$. Since $\mathbb{Z}\left[G_{2}\right] \rightarrow \mathbb{K}\left(G_{2}^{\prime}\right)(t)=\mathbb{K}\left(G_{2}\right)$ is injective it follows that $\varphi(P): \mathbb{Z}\left[G_{2}\right]^{s} \rightarrow \mathbb{Z}\left[G_{2}\right]^{r}$ is injective. By Proposition 6.4 the map $P: \mathbb{Z}\left[G_{1}\right]^{s} \rightarrow \mathbb{Z}\left[G_{1}\right]^{r}$ is injective as well. Since $\mathbb{K}\left(G_{1}^{\prime}\right)(t)=\mathbb{K}\left(G_{1}\right)$ is flat over $\mathbb{Z}\left[G_{1}\right]$ it follows that $P: \mathbb{K}\left(G_{1}^{\prime}\right)(t)^{s} \rightarrow$ $\mathbb{K}\left(G_{1}^{\prime}\right)(t)^{r}$ is injective.

If $\varphi(P)$ is invertible over the skew field $\mathbb{K}\left(G_{2}^{\prime}\right)(t)$, then $r=s$. But an injective homomorphism between vector spaces of the same dimension over a skew field is in fact an isomorphism. This shows that $P$ is invertible over $\mathbb{K}\left(G_{1}^{\prime}\right)(t)$.

Proposition 6.4. If $G_{1}$ is locally indicable, and if $\mathbb{Z}\left[G_{1}\right]^{s} \rightarrow \mathbb{Z}\left[G_{1}\right]^{r}$ is a map such that $\mathbb{Z}\left[G_{1}\right]^{s} \otimes_{\mathbb{Z}\left[G_{1}\right]} \mathbb{Z}\left[G_{2}\right] \rightarrow \mathbb{Z}\left[G_{1}\right]^{r} \otimes_{\mathbb{Z}\left[G_{1}\right]} \mathbb{Z}\left[G_{2}\right]$ is injective, then $\mathbb{Z}\left[G_{1}\right]^{s} \rightarrow$ $\mathbb{Z}\left[G_{1}\right]^{r}$ is injective as well.

Proof. Let $K=\operatorname{Ker}\left\{\varphi: G_{1} \rightarrow G_{2}\right\}$. Clearly $K$ is again locally indicable. Note that $\mathbb{Z}\left[G_{1}\right]^{s} \rightarrow \mathbb{Z}\left[G_{1}\right]^{r}$ can also be viewed as a map between free $\mathbb{Z}[K]$-modules. Pick any right inverse $\lambda: G_{2} \rightarrow G_{1}$ of $\varphi$. It is easy to see that $g \otimes h \mapsto g \lambda(h) \otimes 1, g \in$ $G_{1}, h \in G_{2}$ induces an isomorphism

$$
\mathbb{Z}\left[G_{1}\right] \otimes_{\mathbb{Z}\left[G_{1}\right]} \mathbb{Z}\left[G_{2}\right] \rightarrow \mathbb{Z}\left[G_{1}\right] \otimes_{\mathbb{Z}[K]} \mathbb{Z} .
$$

By assumption $\mathbb{Z}\left[G_{1}\right]^{s} \otimes_{\mathbb{Z}[K]} \mathbb{Z} \rightarrow \mathbb{Z}\left[G_{2}\right]^{r} \otimes_{\mathbb{Z}[K]} \mathbb{Z}$ is injective. Since $K$ is locally indicable it follows immediately from [Gersten 1983] or [Howie and Schneebeli 1983] (see also [Strebel 1974] for the case of PTFA groups) that $\mathbb{Z}\left[G_{1}\right]^{s} \rightarrow \mathbb{Z}\left[G_{1}\right]^{r}$ is injective.

By Lemma 6.2 we have now showed that if $\tau\left(M, \alpha_{2}\right) \neq 0$, then $\tau\left(M, \alpha_{1}\right) \neq 0$. Furthermore

$$
\operatorname{deg} \tau\left(M, \alpha_{i}\right)=\operatorname{deg} \alpha_{i}(B)-\operatorname{deg} \alpha_{i}\left(1-g_{k}\right)-\operatorname{deg} \alpha_{i}\left(1-h_{l}\right), \quad i=1,2 .
$$


Theorem 1.3 now follows immediately from Lemma 6.1 and from the following proposition.

Proposition 6.5. Let $P$ be an $r \times r$ matrix over $\mathbb{Z}\left[G_{1}^{\prime}\right]\left[t^{ \pm 1}\right]$. If $\varphi(P)$ is invertible,

$$
\operatorname{deg} P \geq \operatorname{deg} \varphi(P) .
$$

Remark. (1) If $\varphi: R \rightarrow S$ is a homomorphism of commutative rings, and if $P$ is a matrix over $R\left[t^{ \pm 1}\right]$, then clearly

$$
\operatorname{deg} P=\operatorname{deg} \operatorname{det} P \geq \operatorname{deg} \varphi(\operatorname{det} P)=\operatorname{deg} \operatorname{det} \varphi(P)=\operatorname{deg} \varphi(P) .
$$

Similarly, several other results in this paper, e.g. Theorem 3.1 and Lemma 6.1 are clear in the commutative world, but require more effort in our noncommutative setting.

(2) If ( $\left.\mathbb{Z}\left[G_{1}^{\prime}\right],\left\{f \in \mathbb{Z}\left[G_{1}^{\prime}\right] \mid \varphi(f) \neq 0 \in \mathbb{Z}\left[G_{2}^{\prime}\right]\right\}\right)$ has the Ore property, one can give an elementary proof of the proposition by first diagonalizing over $\mathbb{K}\left(G_{2}^{\prime}\right)$ and then over $\mathbb{K}\left(G_{1}^{\prime}\right)$. Since this is not known to be the case, we have to give a more indirect proof.

The following proof is based on arguments in [Cochran 2004] and [Harvey 2006].

Proof of Proposition 6.5. Let $s=\operatorname{deg} \varphi(P)$. Pick a map $f: \mathbb{Z}\left[G_{1}^{\prime}\right]^{s} \rightarrow \mathbb{Z}\left[G_{1}^{\prime}\right]\left[t^{ \pm 1}\right]^{r}$ such that the induced map

$$
\mathbb{K}\left(G_{2}^{\prime}\right)^{s} \rightarrow \mathbb{K}\left(G_{2}^{\prime}\right)\left[t^{ \pm 1}\right]^{r} \rightarrow \mathbb{K}\left(G_{2}^{\prime}\right)\left[t^{ \pm 1}\right]^{r} / \varphi(P) \mathbb{K}\left(G_{2}^{\prime}\right)\left[t^{ \pm 1}\right]^{r}
$$

is an isomorphism. Denote by $0 \rightarrow C_{1} \stackrel{P}{\rightarrow} C_{0} \rightarrow 0$ the complex

$$
0 \rightarrow \mathbb{Z}\left[G_{1}^{\prime}\right]\left[t^{ \pm 1}\right]^{r} \stackrel{P}{\rightarrow} \mathbb{Z}\left[G_{1}^{\prime}\right]\left[t^{ \pm 1}\right]^{r} \rightarrow 0,
$$

and denote by $0 \rightarrow D_{0} \rightarrow 0$ the complex with $D_{0}=\mathbb{Z}\left[G_{1}^{\prime}\right]^{s}$. We have a chain map $D_{*} \rightarrow C_{*}$ given by $f: D_{0} \rightarrow C_{0}$. Denote by $\operatorname{Cyl}\left(D_{*} \stackrel{f}{\rightarrow} C_{*}\right)$ the mapping cylinder of the complexes. We then get a short exact sequence of complexes

$$
0 \rightarrow D_{*} \rightarrow \operatorname{Cyl}\left(D_{*} \stackrel{f}{\rightarrow} C_{*}\right) \rightarrow \operatorname{Cyl}\left(D_{*} \stackrel{f}{\rightarrow} C_{*}\right) / D_{*} \rightarrow 0 .
$$

More explicitly, we get the commutative diagram

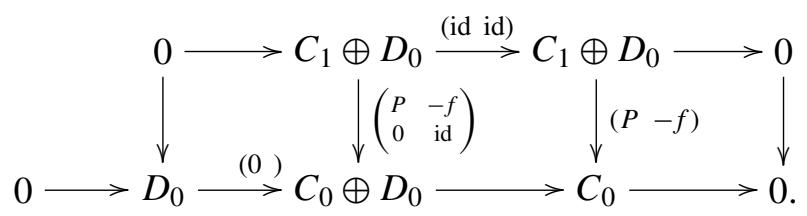


Recall that $\operatorname{Cyl}\left(D_{*} \stackrel{f}{\rightarrow} C_{*}\right)$ and $C_{*}$ are chain homotopic. Using the definition of $f$ we therefore see that

$$
f: H_{0}\left(D_{*} ; \mathbb{K}\left(G_{2}^{\prime}\right)\right) \rightarrow H_{0}\left(\operatorname{Cyl}\left(D_{*} \stackrel{f}{\rightarrow} C_{*}\right), \mathbb{K}\left(G_{2}^{\prime}\right)\right)
$$

is an isomorphism. Since $P$ is invertible over $\mathbb{K}\left(G_{2}^{\prime}\right)(t)$ it follows that

$$
H_{1}\left(\operatorname{Cyl}\left(D_{*} \stackrel{f}{\rightarrow} C_{*}\right) ; \mathbb{K}\left(G_{2}^{\prime}\right)\right)=0 .
$$

It follows from the long exact homology sequence corresponding to the short exact sequence of chain complexes above that

$$
H_{1}\left(\operatorname{Cyl}\left(D_{*} \stackrel{f}{\rightarrow} C_{*}\right) / D_{*} ; \mathbb{K}\left(G_{2}^{\prime}\right)\right)=0 ;
$$

thus the matrix $(P-f)$ is injective over $\mathbb{K}\left(G_{2}^{\prime}\right)$. It follows from Lemma 6.3 that

$$
H_{1}\left(\operatorname{Cyl}\left(D_{*} \stackrel{f}{\rightarrow} C_{*}\right) / D_{*} ; \mathbb{K}\left(G_{1}^{\prime}\right)\right)=0
$$

as well. Again looking at the long exact homology sequence we get that

$$
f: H_{0}\left(D_{*} ; \mathbb{K}\left(G_{1}^{\prime}\right)\right) \rightarrow H_{0}\left(\operatorname{Cyl}\left(D_{*} \stackrel{f}{\rightarrow} C_{*}\right) ; \mathbb{K}\left(G_{1}^{\prime}\right)\right)=H_{0}\left(C_{*} ; \mathbb{K}\left(G_{1}^{\prime}\right)\right)
$$

is an injection. Hence

$$
\begin{aligned}
\operatorname{deg} \varphi(P)=s & =\operatorname{dim}_{\mathbb{K}\left(G_{2}^{\prime}\right)}\left(H_{0}\left(D_{*} ; \mathbb{K}\left(G_{2}^{\prime}\right)\right)\right) \\
& =\operatorname{dim}_{\mathbb{K}\left(G_{1}^{\prime}\right)}\left(H_{0}\left(D_{*} ; \mathbb{K}\left(G_{1}^{\prime}\right)\right)\right) \\
& \leq \operatorname{dim}_{\mathbb{K}\left(G_{1}^{\prime}\right)}\left(H_{0}\left(C_{*} ; \mathbb{K}\left(G_{1}^{\prime}\right)\right)\right)=\operatorname{deg} P .
\end{aligned}
$$

\subsection{Proof of Theorem 1.3 for 3-manifolds with boundary and for 2-complexes.} First let $X$ be a finite connected 2-complex with $\chi(X)=0$. We can give $X$ a CW structure with one 0 -cell. If $n$ denotes the number $n$ of 1 -cells, then $n-1$ equals the number of 2-cells. Now consider the chain complex of the universal cover $\tilde{X}$ :

$$
0 \rightarrow C_{2}(\tilde{X})^{n-1} \stackrel{\partial_{2}}{\rightarrow} C_{1}(\tilde{X})^{n} \stackrel{\partial_{1}}{\rightarrow} C_{0}(\tilde{X})^{1} \rightarrow 0 .
$$

As in Section 6.1 we pick lifts of the cells of $X$ to cells of $\tilde{X}$ to get bases such that if $A_{i}$ denotes the matrix corresponding to $\partial_{i}$, then

$$
A_{1}=\left(1-h_{1}, \ldots, 1-h_{n}\right) .
$$

Note that $\left\{h_{1}, \ldots, h_{n}\right\}$ is a generating set for $\pi_{1}(X)$. Let $l \in\{1, \ldots, n\}$ such that $\phi(l) \neq 0$. The proof of Lemma 6.2 can easily be modified to prove the following.

Lemma 6.6. Denote by $B$ the result of deleting the l-row of $A_{2}$. Then $\tau(X, \alpha) \neq 0$ if and only if $\alpha(B)$ is invertible. Furthermore if $\tau(X, \alpha) \neq 0$, then

$$
\tau(X, \alpha)=\alpha(B) \alpha\left(1-h_{l}\right)^{-1} \in K_{1}\left(\mathbb{K}\left(G_{i}^{\prime}\right)(t)\right) / \pm \alpha_{i}\left(\pi_{1}(X)\right) .
$$


The proof of Theorem 1.3 for closed manifolds can now easily be modified to cover the case of 2-complexes $X$ with $\chi(X)=0$.

Now let $M$ be again a 3-manifold whose boundary consists of a nonempty set of tori. A duality argument shows that $\chi(M)=\frac{1}{2} \chi(\partial(M))=0$. Clearly $M$ is homotopy equivalent to a 2-complex. Reidemeister torsion is not a homotopy invariant but the following lemma still allows us to reduce the case of a 3-manifold with boundary to the case of a 2-complex.

Lemma 6.7 [Turaev 2001, p. 56 and Theorem 9.1]. Let $M$ be a 3-manifold with boundary. Then there exists a 2-complex $X$ and a simple homotopy equivalence $M \rightarrow X$. In particular, if $\alpha: \pi_{1}(X) \cong \pi_{1}(M) \rightarrow \mathrm{GL}(R, d)$ is a representation such that $H_{*}\left(X, R^{d}\right)=0$, then

$$
\tau(M, \alpha)=\tau(X, \alpha) \in K_{1}(R) / \pm \alpha\left(\pi_{1}(M)\right) .
$$

Theorem 1.3 for 3-manifolds with boundary now follows from Theorem 1.3 for 2-complexes $X$ with $\chi(X)=0$.

\section{Harvey's monotonicity theorem for groups}

Let $\pi$ be a finitely presented group and let $(\varphi: \pi \rightarrow G, \phi: \pi \rightarrow \mathbb{Z})$ be an admissible pair for $\pi$. Consider $G^{\prime}=G^{\prime}\left(\phi_{G}\right)$ and pick a splitting $\mathbb{Z} \rightarrow G$ of $\phi_{G}$. As in Section 5.2 we can consider the skew Laurent polynomial ring $\mathbb{K}\left(G^{\prime}\right)\left[t^{ \pm 1}\right]$ together with the $\phi$-compatible representation $\pi \rightarrow \operatorname{GL}\left(\mathbb{K} \ll\left(G^{\prime}\right)\left[t^{ \pm 1}\right], 1\right)$.

Following [Harvey 2006, Definition 1.6] we define $\bar{\delta}_{G}(\phi)$ to be zero if the group $H_{1}\left(\pi, \mathbb{K}\left(G^{\prime}\right)\left[t^{ \pm 1}\right]\right)$ is not $\mathbb{K}\left(G^{\prime}\right)\left[t^{ \pm 1}\right]$-torsion and

$$
\bar{\delta}_{G}(\phi)=\operatorname{dim}_{\mathbb{K}\left(G^{\prime}\right)}\left(H_{1}\left(\pi, \mathbb{K}\left(G^{\prime}\right)\left[t^{ \pm 1}\right]\right)\right)
$$

otherwise. We give an alternative proof for the following result of Harvey [2006, Theorem 2.9].

Theorem 7.1. Let $\pi=\pi_{1}(M)$, where $M$ is a closed 3-manifold. If $\left(\varphi_{1}: \pi \rightarrow G_{1}\right.$, $\left.\varphi_{2}: \pi \rightarrow G_{2}, \phi\right)$ is an admissible triple for $\pi$, then

$$
\begin{array}{ll}
\bar{\delta}_{G_{1}}(\phi) \geq \bar{\delta}_{G_{2}}(\phi) & \text { if }\left(\varphi_{2}, \phi\right) \text { is not initial }, \\
\bar{\delta}_{G_{1}}(\phi) \geq \bar{\delta}_{G_{2}}(\phi)-2 & \text { otherwise. }
\end{array}
$$

Proof. We clearly only have to consider the case that $\bar{\delta}_{G_{2}}(\phi)>0$. We can build $K(\pi, 1)$ by adding $i$-handles to $M$ with $i \geq 3$. It therefore follows that for the admissible pairs $\left(\varphi_{i}: \pi \rightarrow G_{i}, \phi\right)$ we have

$\bar{\delta}_{G_{i}}(\phi)=\operatorname{dim}_{\mathbb{K}\left(G_{i}^{\prime}\right)}\left(H_{1}\left(K_{1}(\pi, 1) ; \mathbb{K}\left(G_{i}^{\prime}\right)\left[t^{ \pm 1}\right]\right)\right)=\operatorname{dim}_{\mathbb{K}\left(G_{i}^{\prime}\right)}\left(H_{1}\left(M ; \mathbb{K}\left(G_{i}^{\prime}\right)\left[t^{ \pm 1}\right]\right)\right)$. 
We combine this equality with Theorem 1.3, Corollary 3.6 and Lemmas 3.2, 4.3, 4.4. The theorem follows now immediately from the observation that $\operatorname{Im}\left\{\pi_{1}(M) \rightarrow\right.$ $\left.G_{i} \rightarrow \operatorname{GL}\left(\mathbb{K}\left(G_{i}^{\prime}\right)\left[t^{ \pm 1}\right], 1\right)\right\}$ is cyclic if and only if $\phi: G_{i} \rightarrow \mathbb{Z}$ is an isomorphism.

This monotonicity result gives in particular an obstruction for a group $\pi$ to be the fundamental group of a closed 3-manifold. For example, Harvey [2006, Example 3.2] shows that as an immediate consequence we get the well-known fact that $\mathbb{Z}^{m}, m \geq 4$, is not a 3 -manifold group.

Remark. In [Friedl and Kim 2005] we considered the case $\pi=\pi_{1}(M)$, where $M$ is a closed 3-manifold. Given an admissible pair $(\varphi: \pi \rightarrow G, \phi)$ we show (under a mild assumption) that $\bar{\delta}_{G}(\phi)$ is even, generalizing [Turaev 1986, p. 141]. In [Friedl and Harvey 2006] it is shown that given $\pi \rightarrow G, G$ locally indicable and amenable, the map

$$
\begin{aligned}
\operatorname{Hom}(G, \mathbb{Z}) & \rightarrow \mathbb{Z} \\
\phi & \mapsto \bar{\delta}_{G}(\phi)
\end{aligned}
$$

defines a seminorm on $\operatorname{Hom}(G, \mathbb{Z})$.

Let $\pi$ be a finitely presented group of deficiency at least one, for example $\pi=$ $\pi_{1}(M)$ where $M$ is a 3-manifold with boundary. Using a presentation of deficiency one we can build a 2-complex $X$ with $\chi(X)=0$ and $\pi_{1}(X)=\pi$. The same proof as the proof of Theorem 7.1 now gives the following theorem of Harvey. (In the case that $\pi=\pi_{1}\left(S^{3} \backslash K\right)$ for $K$ a knot, this was first proved in [Cochran 2004].)

Theorem 7.2 [Harvey 2006, Theorem 2.2]. If $\pi$ is a finitely presented group of deficiency one and if $\left(\varphi_{1}, \varphi_{2}, \phi\right)$ is an admissible triple for $\pi$, then

$$
\begin{array}{ll}
\bar{\delta}_{G_{1}}(\phi) \geq \bar{\delta}_{G_{2}}(\phi) & \text { if }\left(\varphi_{2}, \phi\right) \text { is not initial }, \\
\bar{\delta}_{G_{1}}(\phi) \geq \bar{\delta}_{G_{2}}(\phi)-1 & \text { otherwise. }
\end{array}
$$

\section{Open questions and problems}

Let $M$ be a 3-manifold and $\phi \in H^{1}(M ; \mathbb{Z})$. We propose the following three problems for further study.

(1) If $\left(\varphi: \pi_{1}(M) \rightarrow G, \phi\right)$ is an admissible pair for $\pi_{1}(M)$ and if $\alpha: \pi_{1}(M) \rightarrow$ $\operatorname{GL}(\mathbb{F}, d)$ factors through $\varphi$, does it follow that

$$
\frac{1}{d} \operatorname{deg} \tau(M, \alpha) \leq \operatorname{deg} \tau\left(M, \mathbb{Z}\left[\pi_{1}(M)\right] \rightarrow \mathbb{K}\left(G^{\prime}\right)(t)\right) ?
$$

Put differently, are the Thurston norm bounds of Cochran and Harvey optimal, i.e., at least as good as the Thurston norm bounds of [Friedl and Kim 2006] for any representation factoring through $G$ ? 
(2) In many cases $\operatorname{deg} \tau\left(M, \mathbb{Z}\left[\pi_{1}(M)\right] \rightarrow \mathbb{K}\left(G^{\prime}\right)(t)\right)<\|\phi\|_{T}$, for any admissible pair $\left(\varphi: \pi_{1}(M) \rightarrow G, \phi\right)$. For example this is the case if $K$ is a knot with $\Delta_{K}(t)=1$ and $M=X(K)$. It is an interesting question whether invariants can be defined for any map $\pi_{1}(M) \rightarrow G, G$ a (locally indicable) torsion-free group. For example it might be possible to work with $U(G)$ the algebra of affiliated operators (see [Reich 1998], for instance) instead of $\mathbb{K}(G)$. If such an extension is possible, then it is a natural question whether the Thurston norm is determined by such more general bounds. This might be too optimistic in the general case, but it could be true in the case of a knot complement.

(3) If $(M, \phi)$ fibers over $S^{1}$, the corresponding Alexander polynomial defined over $\mathbb{Z}\left[t^{ \pm 1}\right]$ is monic, that is, the top coefficient is \pm 1 . Because of the high degree of indeterminacy of Alexander polynomials over skew Laurent polynomial rings a corresponding statement is meaningless. Since Reidemeister torsion has a much smaller indeterminacy it is potentially possible to use it to extend the fiberedness obstruction as in [Goda et al. 2005].

\section{Acknowledgment}

The author thanks Stefano Vidussi for pointing out the functoriality of Reidemeister torsion, and Tim Cochran, Shelly Harvey and Taehee Kim for helpful discussions. The author also thanks the referee for many helpful comments.

\section{References}

[Chapman 1974] T. A. Chapman, “Topological invariance of Whitehead torsion”, Amer. J. Math. 96 (1974), 488-497. MR 52 \#11931 Zbl 0358.57004

[Cochran 2004] T. D. Cochran, "Noncommutative knot theory", Algebr. Geom. Topol. 4 (2004), 347-398. MR 2005k:57023 Zbl 1063.57011

[Cochran et al. 2003] T. D. Cochran, K. E. Orr, and P. Teichner, "Knot concordance, Whitney towers and $L^{2}$-signatures", Ann. of Math. (2) 157:2 (2003), 433-519. MR 2004i:57003 Zbl 1044.57001

[Cohn 1985] P. M. Cohn, Free rings and their relations, 2nd ed., London Mathematical Society Monographs 19, Academic Press, London, 1985. MR 87e:16006 Zbl 0659.16001

[Dodziuk et al. 2003] J. Dodziuk, P. Linnell, V. Mathai, T. Schick, and S. Yates, "Approximating $L^{2}$ invariants and the Atiyah conjecture", Comm. Pure Appl. Math. 56:7 (2003), 839-873. Dedicated to the memory of Jürgen K. Moser. MR 2004g:58040 Zbl 1036.58017

[Eisermann 2000] M. Eisermann, "The number of knot group representations is not a Vassiliev invariant”, Proc. Amer. Math. Soc. 128:5 (2000), 1555-1561. MR 2000j:57009 Zbl 0939.57007

[Friedl and Harvey 2006] S. Friedl and S. Harvey, "Non-commutative multivariable Reidemeister torsion and the Thurston norm", preprint, 2006. math.GT/0608409

[Friedl and Kim 2005] S. Friedl and T. Kim, "The parity of the Cochran-Harvey invariants of 3manifolds", preprint, 2005. To appear in Trans. Amer. Math. Soc. math.GT/0510475

[Friedl and Kim 2006] S. Friedl and T. Kim, "The Thurston norm, fibered manifolds and twisted Alexander polynomials”, Topology 45:6 (2006), 929-953. MR MR2263219 Zbl 1105.57009 
[Gersten 1983] S. M. Gersten, "Conservative groups, indicability, and a conjecture of Howie", J. Pure Appl. Algebra 29:1 (1983), 59-74. MR 84m:20035 Zbl 0513.20017

[Goda et al. 2005] H. Goda, T. Kitano, and T. Morifuji, "Reidemeister torsion, twisted Alexander polynomial and fibered knots", Comment. Math. Helv. 80:1 (2005), 51-61. MR 2005m:57008 Zbl 1066.57008

[Harvey 2005] S. L. Harvey, "Higher-order polynomial invariants of three-manifolds giving lower bounds for the Thurston norm”, Topology 44:5 (2005), 895-945. MR 2006g:57019 Zbl 1080.57019

[Harvey 2006] S. L. Harvey, "Monotonicity of degrees of generalized Alexander polynomials of groups and 3-manifolds", Math. Proc. Cambridge Philos. Soc. 140:3 (2006), 431-450. MR 2007c: 57004 Zbl 05025112

[Higman 1940] G. Higman, "The units of group-rings", Proc. London Math. Soc. (2) 46 (1940), 231-248. MR 2,5b JFM 0025.24302

[Howie and Schneebeli 1983] J. Howie and H. R. Schneebeli, "Homological and topological properties of locally indicable groups", Manuscripta Math. 44:1-3 (1983), 71-93. MR 85c:20041 Zbl 0533.20022

[Kirk and Livingston 1999] P. Kirk and C. Livingston, "Twisted Alexander invariants, Reidemeister torsion, and Casson-Gordon invariants”, Topology 38:3 (1999), 635-661. MR 2000c:57010 Zbl 0928.57005

[Leidy and Maxim 2006] C. Leidy and L. Maxim, "Higher-order Alexander invariants of plane algebraic curves", Int. Math. Res. Not. 2006 (2006), ID 12976. MR MR2264729 Zbl 05122980

[Lickorish 1997] W. B. R. Lickorish, An introduction to knot theory, Graduate Texts in Mathematics 175, Springer, New York, 1997. MR 98f:57015 Zbl 0886.57001

[Lin 2001] X. S. Lin, "Representations of knot groups and twisted Alexander polynomials", Acta Math. Sin. (Engl. Ser.) 17:3 (2001), 361-380. MR 2003f:57018 Zbl 0986.57003

[McMullen 2002] C. T. McMullen, "The Alexander polynomial of a 3-manifold and the Thurston norm on cohomology”, Ann. Sci. École Norm. Sup. (4) 35:2 (2002), 153-171. MR 2003d:57044 Zbl 1009.57021

[Milnor 1966] J. Milnor, "Whitehead torsion", Bull. Amer. Math. Soc. 72 (1966), 358-426. MR 33 \#4922 Zbl 0147.23104

[Ranicki 1998] A. Ranicki, High-dimensional knot theory: Algebraic surgery in codimension 2, Springer, New York, 1998. MR 2000i:57044 Zbl 0910.57001

[Reich 1998] H. Reich, Group von Neumann algebras and related algebras, Thesis, University of Göttingen, 1998.

[Rosenberg 1994] J. Rosenberg, Algebraic K-theory and its applications, Graduate Texts in Mathematics 147, Springer, New York, 1994. MR 95e:19001 Zbl 0801.19001

[Stenström 1975] B. Stenström, Rings of quotients: an introduction to methods of ring theory, Grundlehren der Mathematischen Wissenschaften 217, Springer, New York, 1975. MR 52 \#10782 Zbl 0296.16001

[Strebel 1974] R. Strebel, "Homological methods applied to the derived series of groups", Comment. Math. Helv. 49 (1974), 302-332. MR 50 \#7373 Zbl 0288.20066

[Tamari 1957] D. Tamari, "A refined classification of semi-groups leading to generalized polynomial rings with a generalized degree concept", pp. 439-440 in Proceedings of the International Congress of Mathematicians (Amsterdam, 1954), vol. 3, E. P. Noordhoff, Groningen, 1957.

[Thurston 1986] W. P. Thurston, A norm for the homology of 3-manifolds, Mem. Amer. Math. Soc. 339, Amer. Math. Soc., Providence, RI, 1986. MR 88h:57014 Zbl 0585.57006 
[Turaev 1986] V. G. Turaev, "Reidemeister torsion in knot theory”, Uspekhi Mat. Nauk 41:1 (1986), 97-147. In Russian; translated in Russian Math. Surveys 41;1 (1986), 119-182. MR 87i:57009 Zbl 0602.57005

[Turaev 2001] V. Turaev, Introduction to combinatorial torsions, Lectures in Mathematics, ETH Zürich, Birkhäuser, Basel, 2001. MR 2001m:57042 Zbl 0970.57001

[Turaev 2002a] V. Turaev, "A homological estimate for the Thurston norm”, preprint, Univ. Louis Pasteur, Strasbourg, 2002. math.GT/0207267

[Turaev 2002b] V. Turaev, "A norm for the cohomology of 2-complexes", Algebr. Geom. Topol. 2 (2002), 137-155. MR 2002m:57005 Zbl 1014.57002

Received August 31, 2005. Revised December 13, 2005.

\section{STEFAN FRIEDL}

DÉPARTEMENT DE MATHÉMATIQUES

UQAM

C.P. 8888 , SucCursale CENTRE-VILle

Montréal, QC H3C 3P8

CANADA

sfriedl@gmail.com

http://www.labmath.uqam.ca/ friedl/index.html 